\title{
Fall Velocities of Hydrometeors in the Atmosphere: Refinements to a Continuous Analytical Power Law
}

\author{
VITALY I. KHVOROSTYANOV \\ Central Aerological Observatory, Dolgoprudny, Moscow, Russia \\ JUdith A. CURRY \\ School of Earth and Atmospheric Sciences, Georgia Institute of Technology, Atlanta, Georgia
}

(Manuscript received 16 December 2004, in final form 14 June 2005)

\begin{abstract}
This paper extends the previous research of the authors on the unified representation of fall velocities for both liquid and crystalline particles as a power law over the entire size range of hydrometeors observed in the atmosphere. The power-law coefficients are determined as continuous analytical functions of the Best or Reynolds number or of the particle size. Here, analytical expressions are formulated for the turbulent corrections to the Reynolds number and to the power-law coefficients that describe the continuous transition from the laminar to the turbulent flow around a falling particle. A simple analytical expression is found for the correction of fall velocities for temperature and pressure. These expressions and the resulting fall velocities are compared with observations and other calculations for a range of ice crystal habits and sizes. This approach provides a continuous analytical power-law description of the terminal velocities of liquid and crystalline hydrometeors with sufficiently high accuracy and can be directly used in bin-resolving models or incorporated into parameterizations for cloud- and large-scale models and remote sensing techniques.
\end{abstract}

\section{Introduction}

The results of many previous experimental studies of cloud drop and ice crystal terminal velocities have been parameterized in the form of the empirical power laws (e.g., Rogers 1976; Starr and Cox 1985a,b; Pruppacher and Klett 1997; see a detailed review in Mitchell 1996). Such parameterizations are simple and convenient and have been used in many cloud models with both parameterized bulk microphysics (e.g., Starr and Cox 1985a,b; Mitchell 1988, 1994; Cotton and Anthes 1989; Tao et al. 1991; Fu et al. 1995; Krueger et al. 1995; Morrison et al. 2005a,b), and explicit spectral microphysics (e.g., Jensen et al. 1994; Khvorostyanov 1995; Khvorostyanov et al. 2003), in general circulation models (GCMs; e.g., Fowler et al. 1996), and in remote sensing studies (e.g., Sassen 1980; Matrosov and

Corresponding author address: Dr. J. A. Curry, School of Earth and Atmospheric Sciences, Georgia Institute of Technology, Atlanta, GA 30332.

E-mail: curryja@eas.gatech.edu
Heymsfield 2000). However, previous power-law parameterizations of the terminal velocities have been mostly empirical and their dependence on the parameters of the particle and atmosphere were often uncertain, including the temperature-pressure dependence (i.e., height dependence).

Based upon research by Abraham (1970), Beard (1980) found an analytical expression for the relation of the Reynolds number Re and the Best or Davies number $X$ for the drops that served as a platform for the further studies of the terminal velocity $V_{t}$. Böhm (1989, 1992) extended this approach and applied it to both drops and crystals. However, these $\operatorname{Re}-X$ relations and the resulting fall speeds were of algebraic and not of the power-law form. Mitchell (1996, hereafter M96) found a physically based power law for the $\operatorname{Re}-X$ relation and for the terminal velocity $V_{t}$ of hydrometeors for each of four ranges of $X$. M96 formulated this theory in terms of the mass-size and area-size parameters, and compiled a set of these parameters for various crystal habits. Hence, M96 provided an improved physical basis for the parameterization of the terminal velocity of hy- 
drometeors. This approach was developed further by Heymsfield and Iaquinta (2000), who applied it for evaluation of $V_{t}$ for bullet rosettes and columns in cirrus.

The research of M96 was extended by Khvorostyanov and Curry (2002, hereafter KC02), who improved upon the discontinuous nature of the M96 parameterization with an analytical power-law-type representations for the $\mathrm{Re}-X$ relation and for fall velocities with coefficients as continuous functions of $X$ or particle diameter $D$ for the entire size range. $\mathrm{KC} 02$ applied these equations for evaluation of the fall speeds and found good agreement with experimental data for droplets and several crystal habits.

Mitchell and Heymsfield (2005, hereafter MH05) pointed out that application of $\mathrm{KC} 02$ scheme for some crystal habits that reach large sizes leads to an overestimation of the fall speeds, especially for large aggregates, and better agreement is reached with alternate values of the parametric constants recommended by Böhm $(1989,1992)$. In addition, MH05 showed that even using the parameters for the crystals, the fall speeds of large crystal aggregates evaluated with $\mathrm{KC} 02$ scheme are overestimated and suggested improving this scheme by introducing some empirical corrections to the $\mathrm{KC} 02$ power-law coefficients.

In this paper, we develop further our previous scheme, $\mathrm{KC} 02$. The $\mathrm{KC} 02$ scheme is improved by two modifications: (i) the use of the constants for crystals recommended by Böhm, and (ii) with account for the turbulent corrections to Reynolds number and to the coefficients of the power law. No further empirical corrections are needed and the fall speeds of both droplets and crystals (including large aggregates and hail) can be evaluated with this scheme with sufficiently high accuracy.

The structure of this paper is as follows. The basic equations are given in section 2 , and the turbulence corrections are derived in section 3. Asymptotic values of the coefficients of power laws for the $\mathrm{Re}-X$ relation and for the fall speeds are obtained in section 4. Section 5 is devoted to the corrections of terminal velocities for temperature and pressure. In section 6 , the expressions for $\mathrm{Re}-X$ relation and drag coefficients are evaluated for drops and crystals with special attention paid to the turbulent corrections. Applications of this scheme are described for various crystal habits from relatively small crystals to aggregates and hail.

\section{Basic equations}

The general expression for terminal velocity of a falling body, $V_{t}$, is given by equating the drag force $F_{D}$ to the difference of the gravitational force $m g=\rho_{b} \mathrm{v}_{b} g$ and the buoyancy force $F_{b}=\rho_{F} \mathrm{v}_{b} g$ :

$$
V_{t}=\left[\frac{2\left(\left|m g-F_{b}\right|\right)}{\rho_{F} A C_{D}}\right]^{1 / 2}=\left(\frac{2 g v_{b}}{C_{D} A}\left|\frac{\rho_{b}}{\rho_{F}}-1\right|\right)^{1 / 2},
$$

where the following parameters are defined for the falling body: maximum dimension $D$, mass $m$, density $\rho_{b}$, projected area $A$, and volume $v_{b}$. The fluid in which the body is falling is defined by its density $\rho_{F}$, dynamic viscosity $\eta$, and kinematic viscosity $\nu=\eta / \rho_{F}$. The parameter $g$ is the gravitational acceleration and $C_{D}$ is the drag coefficient. The notation $|.$.$| refers to absolute$ value, since here we consider the positive differences, that is, falling bodies if they are denser than the fluid $\left(\rho_{b}>\rho_{F}\right)$ and rising bodies in the opposite case $\rho_{b}<\rho_{F}$. For hydrometeors in the air, $\rho_{b} \gg \rho_{F}$, and the term -1 under the absolute value sign can be neglected (note that for sand particles in the ocean or for the dropsondes or radiosondes in the air, $\rho_{b} / \rho_{F} \sim 1$, and the -1 term should be retained).

The primary challenge in evaluating (2.1) is to determine the value of $C_{D}$. Following Abraham (1970), the drag coefficient is represented as a function of the Reynolds number ( $\mathrm{Re}$ ),

$$
C_{D}=C_{0}\left(1+\delta_{0} / \mathrm{Re}^{1 / 2}\right)^{2},
$$

where $C_{0}$ is the drag coefficient for the potential flow without friction around the assembly of the body and its boundary layer, $\delta_{0}$ is the boundary layer depth, and

$$
\operatorname{Re}=\frac{V_{t} D}{\nu}=\frac{V_{t} D \rho_{F}}{\eta} .
$$

The Davies (or Best) number $X$ is related to Re by

$$
\begin{aligned}
X & =C_{D} \operatorname{Re}^{2} \\
& =\frac{2\left|m g-F_{b}\right| \rho_{F} D^{2}}{A \eta^{2}}=\frac{2 \mathrm{v}_{b}\left|\rho_{b}-\rho_{F}\right| g D^{2}}{A \rho_{F} \nu^{2}} .
\end{aligned}
$$

Beard $(1980)$ and Böhm $(1989,1992)$ found an analytical $\operatorname{Re}-X$ relation by substituting (2.2) into (2.4a),

$$
\operatorname{Re}=\left(\delta_{0}^{2} / 4\right)\left[\left(1+C_{1} X^{1 / 2}\right)^{1 / 2}-1\right]^{2},
$$

where $C_{1}=4 /\left(\delta_{0}^{2} C_{0}^{1 / 2}\right)$.

Based upon (2.5), M96 expressed the relation between $\operatorname{Re}$ and $X$ as

$$
\operatorname{Re}(X)=a_{\mathrm{Re}} X^{b \mathrm{Re}} .
$$

M96 found the best numerical fits for $a_{\mathrm{Re}}, b_{\mathrm{Re}}$ in four $X$ intervals for $10^{-2}<X<10^{8}$. KC02 extended this work by determining expressions of $a_{\mathrm{Re}}$ and $b_{\mathrm{Re}}$ as continu- 
ous analytical functions of $X$ over the entire range of its variation as

$$
\begin{aligned}
a_{\mathrm{Re}}(X) & =\frac{\operatorname{Re}(X)}{X^{b_{\mathrm{Re}}}}=\frac{\delta_{0}^{2}}{4} \frac{\left[\left(1+C_{1} X^{1 / 2}\right)^{1 / 2}-1\right]^{2}}{X^{b_{\mathrm{Re}}}}, \\
b_{\mathrm{Re}}(X) & =X \frac{\operatorname{Re}^{\prime}}{\operatorname{Re}} \\
& =\frac{C_{1} X^{1 / 2}}{2\left[\left(1+C_{1} X^{1 / 2}\right)^{1 / 2}-1\right]\left(1+C_{1} X^{1 / 2}\right)^{1 / 2}},
\end{aligned}
$$

where the prime means the derivative by $X$. Expressing the drag coefficient from (2.4a) as $C_{D}=X / \mathrm{Re}^{2}$ and substituting (2.6) into (2.1), KC02 obtained the following expression for the terminal velocity:

$$
\begin{aligned}
V_{t} & =a_{\operatorname{Re}} \nu^{1-2 b_{\operatorname{Re}}}\left(\frac{2\left|m g-F_{b}\right|}{\rho_{F} A}\right)^{b_{\mathrm{Re}}} D^{2 b_{\mathrm{Re}}-1} \\
& =a_{\operatorname{Re}} \nu^{1-2 b_{\operatorname{Re}}}\left(\frac{2 \mathrm{v}_{b} g}{A}\left|\frac{\rho_{b}}{\rho_{F}}-1\right|\right)^{b_{\mathrm{Re}}} D^{2 b_{\mathrm{Re}}-1} .
\end{aligned}
$$

If the objects can be characterized by the massdimension $m-D$ and area-dimension $A-D$ relations,

$$
m=\alpha D^{\beta}, \quad A=\gamma D^{\sigma},
$$

as, for example, in M96 for hydrometeors, substituting (2.11) into (2.9) and neglecting $F_{b}$, yields a power-lawlike representation for the fall velocity as a function of $D$,

$$
\begin{aligned}
& V_{t}=A_{v} D^{B_{v}} \\
& A_{v}=a_{\operatorname{Re}} \rho_{a}^{b_{\operatorname{Re}}-1} \eta^{1-2 b_{\operatorname{Re}}}\left(\frac{2 \alpha g}{\gamma}\right)^{b_{\mathrm{Re}}} \\
& B_{v}=b_{\operatorname{Re}}(\beta-\sigma+2)-1
\end{aligned}
$$

Equation (2.14) is the same as in M96, where the coefficients $a_{\mathrm{Re}}, b_{\mathrm{Re}}$ were fitted in the four $X$ intervals, and (2.13) is written in the form that allows easier derivation of the altitude correction for $V_{t}$. In the approach described here, following $\mathrm{KCO} 2$, we employ a continuous power-law representation of the terminal velocity over the entire particle size range, which is achieved by using continuous coefficients $a_{\mathrm{Re}}, b_{\mathrm{Re}}$ defined by (2.7), (2.8). The values $\delta_{0}=9.06, C_{0}=0.292$ were used in $\mathrm{KC} 02$ for the drops and crystals yielding a good agreement with experiment for the drops and a reasonable agreement for the relatively small crystal fractions.

MH05 noted that application of the $\mathrm{KC} 02$ scheme for single crystals becomes better with use of the values $\delta_{0}=5.83, C_{0}=0.6$ recommended by Böhm (1989, 1992) for crystals; however even with these parameters application for crystal aggregates of $\mathrm{KC} 02$ scheme based on Böhm's relation (2.5) may lead to overestimation of the fall speeds by up to $30 \%$. To remove this discrepancy, MH05 modified Böhm's relation (2.5) for applications for aggregates by adding an additional empirical term

$$
\operatorname{Re}_{\mathrm{MH}}(X)=\operatorname{Re}_{\mathrm{BB}}(X)-a_{0} X^{b_{0}},
$$

where $\mathrm{Re}_{\mathrm{BB}}$ is Beard-Böhm's $\mathrm{Re}-X$ relation $(2.5), a_{0}=$ $1.7 \times 10^{-3}, b_{0}=0.8$, and then MH05 accordingly modified the $\mathrm{KC0} 2$ power-law relations (2.6)-(2.8).

This modification allowed $\mathrm{MH} 05$ to reach a better agreement with observed fall speeds for crystal aggregates. Note that such modification is valid for a limited range of $X$, since $\mathrm{Re}$ in (2.15) reach a maximum at $X \sim$ $10^{9}$ and then decreases with growing $X$; an asymptotic analysis of (2.15) shows that $\mathrm{Re}$ becomes negative at $X$ $\geq\left(C_{0}^{1 / 2} a_{0}\right)^{-1 /\left(b_{0}-1 / 2\right)} \sim 4 \times 10^{9}$. Since even for the largest snow aggregates $X \leq 10^{7}$ (see Fig. 2 in MH05), the approach by MH05 is valid with a good accuracy for the entire size range of snow aggregates. The other falling bodies may reach greater values of $X \geq 10^{9}$. For instance, for the large hailstones $2.5-10 \mathrm{~cm}$, Re reaches 3 $\times 10^{4}-3.2 \times 10^{5}$, and $X$ reaches $10^{9}-10^{11}$ [see Fig. 3 in Böhm (1989) and Fig. 9 in M96]. Comparable values are observed for the dropsondes. Such objects may require different corrections for the large sizes.

It is shown below that an empirical modification is not necessary if appropriate turbulent corrections are introduced into the $\mathrm{KC} 02$ scheme, which allows continuous representation of $\operatorname{Re}(X)$ over the entire $X$ range up to the values characteristic for largest hailstones with $D \sim 5-10 \mathrm{~cm}$, and even up to the larger values $X>10^{11}\left(\mathrm{Re}>3 \times 10^{5}\right)$ characteristic for dropsondes and radiosondes.

\section{Turbulence corrections}

When Re approaches $\sim 10^{3}, C_{D}$ begins to increase, which is caused by the effects of turbulence in the flow. Böhm (1992) suggested an analytical parameterization of $C_{D}$ in this transitional regime, whereby the turbulent drag coefficient $C_{D t}$ is related to the $C_{D l}$ in a laminar flow using an interpolation function $\psi(X)$,

$$
\begin{aligned}
C_{D t}(X) & =C_{D l}(X) \psi^{-1}(X), \\
\psi(X) & =\frac{1+\left(X / X_{0}\right)^{k}}{1+C_{t}\left(X / X_{0}\right)^{k}} .
\end{aligned}
$$

Limiting values of $\psi$ are $\psi=1$ at small $X \ll X_{0}$, and $\psi^{-}=C_{t}^{-1}$ at large $X \gg X_{0}$. Böhm (1992) used fixed values $C_{t}=1.6, k=2, X_{0}=6.7 \times 10^{6}$ for drops and $X_{0}=2.8 \times 10^{6}$ for crystals. The value of $C_{t}=1.6$ characterizes an observed in some experiments increase of $60 \%$ in the drag coefficient caused by turbulence (see review in Böhm 1992), while Mitchell (1996) used 
$C_{t}=1.3$ to better conform to the data from Knight and Heymsfield (1983).

Here we generalize Böhm's parameterization. First, substitute (3.1) into (2.4a),

$$
\operatorname{Re}_{t}(X)=X^{1 / 2} / C_{D t}^{1 / 2}=\operatorname{Re}_{l}(X)[\psi(X)]^{1 / 2},
$$

where $\mathrm{Re}_{t}$ is the turbulent Reynolds number and $\mathrm{Re}_{l}$ is the "laminar" Reynolds number described by BeardBöhm's relation (2.5). Following $\mathrm{KC} 02, \mathrm{Re}_{l}$ can be formulated as a power law analogous to (2.6)-(2.8), and then (3.3) provides an analytical representation of the $\operatorname{Re}-X$ relation corrected for turbulence. Substituting (3.3) into (2.8) for $b_{\mathrm{Re}}$, we obtain the coefficient $b_{\mathrm{Re}, t}$ corrected for turbulence,

$$
b_{\mathrm{Re}, t}(X)=X \operatorname{Re}_{t}^{\prime}(X) / \operatorname{Re}_{t}(X)=b_{\mathrm{Re}, l}(X)+\Delta b_{\mathrm{Re}, t}(X),
$$

where $b_{\mathrm{Re}, l}=X \operatorname{Re}_{l}^{\prime} / \operatorname{Re}_{l}$ is the value of $b_{\mathrm{Re}}$ for the laminar flow defined in (2.8) and $\Delta b_{\mathrm{Re}, t}$ is the turbulent correction

$$
\Delta b_{\mathrm{Re}, t}=\frac{X}{2} \frac{\psi^{\prime}}{\psi}=-\frac{k\left(C_{t}-1\right) z^{k}}{2\left(1+z^{k}\right)\left(1+C_{t} z^{k}\right)},
$$

where $z=X / X_{0}$. Substituting (3.3) into (2.7) we find for $a_{\mathrm{Re}}$

$$
a_{\mathrm{Re}, t}(X)=\frac{\operatorname{Re}_{t}(X)}{X^{b_{\mathrm{Re}, t}}}=a_{\mathrm{Re}, l}(X) \xi_{t}(X),
$$

where $a_{\mathrm{Re}, l}$ is the value for the laminar flow defined by (2.7) and $\xi_{t}$ is its turbulent correction

$$
a_{\mathrm{Re}, l}=\frac{\operatorname{Re}_{l}(X)}{X^{b_{\mathrm{Re}, l}}}, \quad \xi_{t}(X)=\frac{[\psi(X)]^{1 / 2}}{X^{\Delta b_{\mathrm{Re}, t}}} .
$$

The corrections $\Delta b_{\mathrm{Re}, t}$ and $\xi_{t}$ are described in more detail later in sections 4 and 6 .

\section{Asymptotic values}

The analytical representation of $a_{\mathrm{Re}}, b_{\mathrm{Re}}, A_{v}, B_{v}$ as given above allows for straightforward estimate of the asymptotic limits, which is useful for estimation of the limiting behavior of the fall velocities. It is seen from (2.7), (2.8) that the asymptotic values of $a_{\mathrm{Re}}, b_{\mathrm{Re}}$ are reached at $C_{1} X^{1 / 2} \ll 1$, and $C_{1} X^{1 / 2} \gg 1$. Thus, a scaling Best parameter $X_{\mathrm{sc}}$ can be introduced from the condition $C_{1} X_{\mathrm{sc}}^{1 / 2}=1$, or $X_{\mathrm{sc}}=1 / C_{1}^{2}=\delta_{0}^{4} C_{0} / 16$, corresponding to $X_{\mathrm{sc}} \approx 122$ for drops (rigid spheres) and $X_{\mathrm{sc}} \approx 43$ for crystals with values of $C_{0}$ and $\delta_{0}$ cited above. The value of $X_{\mathrm{sc}}$ separates the two regimes for small and large particles or the regimes of potential $\left(X \ll X_{\mathrm{sc}}\right)$ and aerodynamical $\left(X \gg X_{\mathrm{sc}}\right)$ flows. The scheme for $V_{t}$ above is based upon Abraham's (1970) theory of the boundary layer, which should be valid for $\mathrm{Re} \gg 1$. Beard (1980) showed that Abraham's theory can be extended to the small particle limit, which reduces to Stokes law for drops and appears to be valid with high accuracy for $\operatorname{Re} \ll 1$ also. The analytical schemes in $\mathrm{KC} 02$ and this work allow obtaining analytical asymptotics both at $X \ll X_{\mathrm{sc},}$, and $X \gg X_{\mathrm{sc} \text {. }}$.

For $X \ll X_{\mathrm{sc} \text {, }}$ the asymptotic values for $a_{\mathrm{Re}, l}, b_{\mathrm{Re}, l}$ are obtained from (2.7) and (2.8) by expanding into the power series

$$
b_{\mathrm{Re}, l}(X)=1, \quad a_{\mathrm{Re}, l}(X)=1 /\left(C_{0} \delta_{0}^{2}\right) .
$$

This yields $a_{\mathrm{Re}, l}=0.0417$ for drops and $a_{\mathrm{Re}, l}=0.049$ for crystals, in a good agreement with Mitchell's (1996) fit $a_{\mathrm{Re}}=0.0439, b_{\mathrm{Re}}=0.97$ in the range of smallest $X=$ 0.01 to 10 . Since $\psi(X)=1$ at $X \ll X_{\mathrm{sc}}$, the limits of $a_{\mathrm{Re}, t}$, $b_{\mathrm{Re}, t}$ are the same.

M96 and then $\mathrm{KC} 02$ found the Stokes limit of $V_{t}$ for small particles that can be obtained by substitution of asymptotic values for $a_{\mathrm{Re}}, b_{\mathrm{Re}}$ from (4.1) into (2.12)(2.14), which yields

$$
\begin{aligned}
V_{t}(r) & =A_{v} D^{B_{v}}=A_{v r} r^{B_{v}}, \\
A_{v} & =\frac{4 \rho_{w} g}{3 \delta_{0}^{2} C_{0} \eta}, \quad A_{v r}=\frac{16 \rho_{w} g}{3 \delta_{0}^{2} C_{0} \eta}, \quad B_{v}=2 .
\end{aligned}
$$

Using $\delta_{0}=9.06, C_{0}=0.292$ gives $A_{v r}=1.2 \times 10^{6} \mathrm{~cm}^{-1}$ $\mathrm{s}^{-1}$, that is, the Stokes constant for the drops, while (4.2) yields the Stokes law $V_{t} \sim r^{2}$. These expressions are used in section 5 for verification of the temperature and pressure dependence of the fall speeds for small particles.

In the opposite limit, $X \gg X_{\mathrm{sc}}$, it follows from (2.7), (2.8) that

$$
b_{\mathrm{Re}, l}=1 / 2, \quad a_{\mathrm{Re}, l}=1 / C_{0}^{1 / 2},
$$

resulting in $a_{\mathrm{Re}, l}=1.86$ for drops and 1.29 for crystals. The turbulent corrections influence the behavior of $a_{\mathrm{Re}, t}, b_{\mathrm{Re}, t}$ in the region of large $X$, which is determined by the major change of the function $\psi(X)$ around $X_{0}$. With the choice $X_{0}=2.8 \times 10^{6}$ for crystals as in Böhm (1992), evaluation of $\psi(X)$ and of $a_{\mathrm{Re}, t}(X), b_{\mathrm{Re}, t}(X)$ described in section 6 shows that this region extends from $X_{1 t} \sim 3 \times 10^{4}\left(\operatorname{Re} \sim 2 \times 10^{2}\right)$ to $X_{2 t} \sim 10^{8}(\mathrm{Re} \sim$ $\left.10^{4}\right)$. It is seen from (3.5) that the turbulent correction $\Delta b_{\mathrm{Re}, t}$ tends to zero at asymptotically large $X$ as $1 / X^{2}$, and (3.4) shows that turbulence does not effect $b_{\mathrm{Re}, t}$ at very large $X>X_{2 t}$ and its asymptotic value coincides with that for the laminar flow. Equation (3.7) shows that since at sufficiently large $X>X_{2 t}, \Delta b_{\mathrm{Re}, t} \rightarrow 0$, then 
$\xi(X) \rightarrow[\psi(X)]^{1 / 2} \rightarrow 1 / C_{t}^{1 / 2}$, so that the asymptotic values of $a_{\mathrm{Re}, t}$ and $b_{\mathrm{Re}, t}$ are

$$
b_{\mathrm{Re}, t}=1 / 2, \quad a_{\mathrm{Re}, t}=1 /\left(C_{0}^{1 / 2} C_{t}^{1 / 2}\right)=1.02
$$

(with $C_{t}=1.6$ ), and asymptotic relations between the coefficients with and without turbulent corrections are

$$
b_{\mathrm{Re}, t}=b_{\mathrm{Re}, l}, \quad a_{\mathrm{Re}, t}=a_{\mathrm{Re}, l} / C_{t}^{1 / 2} .
$$

Using $C_{t}=1.6$, as in Böhm (1992), (4.5) gives the asymptotic values $a_{\mathrm{Re}, t}=1.46$ and 1.02 for drops and crystals, respectively. With $C_{t}=1.3$, as in M96, we obtain $a_{\mathrm{Re}, t}=1.57$ and 1.09 for drops and crystals, respectively. Our analytical limiting value $b_{\mathrm{Re}, t}=1 / 2$ in (4.5) is very close to M96 fit $b_{\mathrm{Re}}=0.499$ at $1.56 \times 10^{5}<X$ $<10^{8}$ as well as our limit of $a_{\mathrm{Re}, t}=1.09$ with $C_{t}=1.3$ for the crystals compared to Mitchell's fit $a_{\mathrm{Re}}=1.0865$. Thus, (4.5) can be used with a good accuracy to estimate the asymptotic regimes of the fall velocities. The Re $-X$ relation reduces for $X \gg X_{\mathrm{sc}}$ and $C_{t}=1.6$ to a simple square root law

$$
\operatorname{Re}(X)=\frac{1}{\left(C_{0} C_{t}\right)^{1 / 2}} X^{1 / 2}=1.02 X^{1 / 2} .
$$

So, at large $X>X_{2 t}$, the turbulence correction with $C_{t}=1.6$ leads to a decrease by $1 / C_{t}^{1 / 2}=1 / 1.265$ in the coefficient $a_{\mathrm{Re}, t}$ in the power law (2.7) and, according to (2.12), (2.13), in $V_{t}$. This decrease in $V_{t}$ by $\sim 26 \%$ removes the tendency noted by $\mathrm{MH} 05$ of the $\mathrm{KC} 02$ scheme to overestimate $\operatorname{Re}(X)$ and $V_{t}$ by up to $30 \%$ for large crystals and aggregates, and makes $\mathrm{Re}$ and velocities calculated with this scheme much closer to those observed without empirical corrections as used in MH05. Hence, these equations with turbulent corrections yield description for the large aggregates equivalent to MH05, and in addition, can be used up to very large $X>10^{12}$ and $\operatorname{Re}>10^{6}$, in particular for large hailstones, which is illustrated in section 6 .

Turbulence does not change the asymptotic index $b_{\mathrm{Re}, t}$, which determines the asymptotic behavior of $\operatorname{Re}(X)$ in (2.6) and of $V_{t}(D)$ in (2.12). Note, however, that the correction $\Delta b_{\mathrm{Re}}$ and the coefficient $b_{\mathrm{Re}}$ have a minimum at moderately large $X \sim(2-3) \times 10^{6}$, corresponding to $D \sim 0.3-2 \mathrm{~cm}$ for the large aggregates and hail. The larger values of $X \geq X_{2 t} \sim 10^{8}$ may not be reached by many crystal habits due to limitations to their sizes, and their asymptotic behavior is determined by the intermediate region of transition to turbulent regime $X_{1 t}<X<X_{2 t}$ as illustrated in section 6. Also, at $\operatorname{Re}>10^{5}$ or $X>10^{10}$, the asymptotics (4.5) may become invalid as the drag crisis is encountered, which is considered in section $6 \mathrm{~b}$.

\section{Corrections for temperature and pressure}

The equations for $V_{t}$ were derived above for fixed values of pressure $p$, and temperature $T$. As seen from (2.12)-(2.14), there are two kinds of dependencies of $V_{t}$ on $p$ and $T: 1)$ directly via $\eta$ and $\rho_{a}$ in $A_{v}(p, T)$, and 2) via dependence of $a_{\mathrm{Re}}, b_{\mathrm{Re}}$ on $X$, which in turn depends on $p, T$.

Consider first the direct $\eta$ and $\rho_{a}$ dependence in $A_{v}(p, T)$, which is defined in (2.13),

$$
V_{t} \sim A_{v}(p, T) \sim \eta^{\left(1-2 b_{\mathrm{Re}}\right)} \rho_{a}^{\left(b_{\mathrm{Re}}-1\right)} .
$$

The value of $\eta$ is almost independent of $p$ (Pruppacher and Klett 1997, hereafter PK97), and its temperature dependence is $\eta(T)=\eta_{0} \times \varphi(T)$, where $\eta_{0}=1.718 \times$ $10^{-4}$ poise is the dynamic viscosity at $0^{\circ} \mathrm{C}$, and

$$
\begin{aligned}
& \varphi(T)=1+0.00285 T_{c}-6.9 \times 10^{-6} T_{c}^{2}, T_{c}<0^{\circ} \mathrm{C}, \\
& \varphi(T)=1+0.00285 T_{c}, T_{c}>0{ }^{\circ} \mathrm{C}, \text { and }
\end{aligned}
$$

$T_{c}=T-273.15$ is the temperature in degrees Celsius (PK97). Using these expressions with the equation of state $\rho_{a}=p /\left(R_{a} T\right)$, with $R_{a}$ being the specific gas constant for the dry air, and substituting into (5.2), we obtain an expression for the velocity $V_{t}$ at a height with $p, T$ to its value $V_{t 0}$ a height with $p=p_{0}$, and $T=T_{0}$ :

$$
\begin{aligned}
V_{t}(p, T) & =C_{p T} V_{t 0} \\
C_{p T} & =\left[\frac{\eta(T)}{\eta\left(T_{0}\right)}\right]^{1-2 b_{\mathrm{Re}}}\left[\frac{\rho_{a}\left(p_{0}, T_{0}\right)}{\rho_{a}(p, T)}\right]^{1-b_{\mathrm{Re}}} \\
& =[\varphi(T)]^{1-2 b_{\mathrm{Re}}}\left(\frac{p_{0}}{p} \frac{T}{T_{0}}\right)^{1-b_{\mathrm{Re}}} .
\end{aligned}
$$

The two asymptotic cases follow from (5.3c) and (4.1), (4.5). For small sizes, the index $b_{\mathrm{Re}}=1$, and $1-2 b_{\mathrm{Re}}$ $=-1,1-b_{\mathrm{Re}}=0$, then the pressure or air density dependencies in $(5.3 \mathrm{~b}, \mathrm{c})$ vanish, and $V_{t}$ depends on the temperature via weak function as

$$
C_{p T}=[\varphi(T)]^{-1}=\left[\eta(p, T) / \eta_{0}\right]^{-1},
$$

and its decrease from $0^{\circ}$ to $-40^{\circ} \mathrm{C}$ is $\sim 12 \%$. This is confirmed by comparison of (5.4) to the Stokes limit for the small particles (4.2), (4.3), and to this limit in Beard (1980) where also $V_{t} \sim \eta^{-1}$, which indicates the validity of this $C_{p T}$ limit in the viscous regime.

For large sizes, according to (4.5), $1-2 b_{\mathrm{Re}}=0$, and $1-b_{\mathrm{Re}}=0.5$, then the dependence on $\varphi(T)$ or dy- 
namic viscosity $\eta$ vanishes, and the $p, T$ dependencies are described by the square root law by $\rho_{a}$ or by $p, T$ as

$$
\begin{aligned}
V_{t}(p, T) & =c_{p T \infty} V_{t 0}, \\
c_{p T \infty} & =\left[\frac{\rho\left(p_{0}, T_{0}\right)}{\rho(p, T)}\right]^{1 / 2}=\left(\frac{p_{0}}{p} \frac{T}{T_{0}}\right)^{1 / 2} .
\end{aligned}
$$

The dependence (5.5) can be obtained directly from (2.4) assuming $C_{D}=$ const, coincides with the corresponding limit in Beard (1980) and is typical of the aerodynamic limit used in many numerical models for precipitating species (e.g., Kessler 1969; Lin et al. 1983; Rutledge and Hobbs 1983; Fowler et al. 1996). A comparison of (5.4) and (5.5) shows that the temperature and pressure dependence of the fall speeds is substantially different for the small $(r \leq \sim 30-40 \mu \mathrm{m}$ or $D \leq$ 60-80 $\mu \mathrm{m})$ and large particles: the velocities of the smaller fraction increase with increasing height much slower than those of the large fractions. Hence, the difference of fall speeds between the small and large fractions increases with height. This increase between the surface $\left(1000 \mathrm{hPa}\right.$ and $\left.0^{\circ} \mathrm{C}\right)$ and, for example, a height with $p=300 \mathrm{hPa}, T=-40^{\circ} \mathrm{C}$ is $\sim 12 \%$ for small fraction (due to $\varphi^{-1}$ ) and $170 \%$ for the large fraction according to (5.5). Accounting for this effect will lead to acceleration of coagulation and accretion with growing altitude, which depends on differential fall speeds between large and small particles.

The second kind of $V_{t}$ variations with $p$ and $T$ due to changes in $X$ and $a_{\mathrm{Re}}, b_{\mathrm{Re}}$ can be estimated using the relations

$$
\delta a_{\operatorname{Re}} \sim \frac{\partial a_{\mathrm{Re}}}{\partial X} \delta X, \quad \delta b_{\mathrm{Re}} \sim \frac{\partial b_{\mathrm{Re}}}{\partial X} \delta X .
$$

For estimates of $X$ variations, (2.4b) can be rewritten using $m-D, A-D$ relations (2.11), and equations for $\rho_{a}(T)$ and $\eta(T)$. Using Eqs. (2.7), (2.8) for $a_{\mathrm{Re}}, b_{\mathrm{Re}}$, we performed both analytical and numerical evaluation of these variations of $V_{t}$, which are 1-3 orders of magnitude smaller than the direct dependence described above and therefore can be neglected.

So, (5.3a)-(5.3c) describe the dependence of $V_{t}$ on temperature and pressure with sufficient precision and yield correct limits in both viscous and aerodynamic regimes for small and precipitating fractions, respectively. The accuracy of (5.3a)-(5.3c) for $C_{p T}$ correction is illustrated in section $6 \mathrm{f}$ by a comparison with Beard's (1980) parameterization and with the direct numerical calculations of $V_{t}$ at various heights.

\section{Results}

In this section, this scheme is compared to the other methods and to the experimental data. The features of

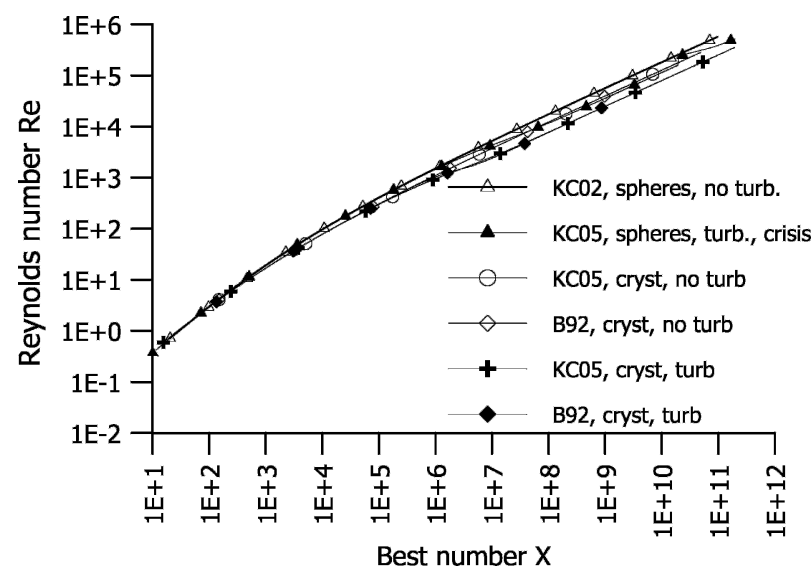

FIG. 1. $\mathrm{Re}-X$ relation calculated using $\mathrm{KC} 02$ with $C_{0}, \delta_{0}$ for rigid spheres or drops without turbulent correction ( $\mathrm{KC} 02$; open triangles), for spheres with corrections for turbulence and drag crisis (KC05, solid triangles), with $C_{0}, \delta_{0}$ for crystals without turbulent correction (KC05, open circles), and with this correction ( $\mathrm{KC05}$, crosses), compared to Böhm (1992) without turbulent correction (B92; open diamonds) and with correction (B92; solid diamonds). The curves from MH05 (not shown) are very close to the corresponding curves B92 and $\mathrm{KC} 05$.

Re $-X$ relation, drag coefficient, and of the coefficients $a_{\mathrm{Re}}$ and $b_{\mathrm{Re}}$ are illustrated and this method is applied for evaluation of fall velocities of various crystal habits, which allows estimating its accuracy. For brevity, a modification of the $\mathrm{KC} 02$ scheme performed in this paper is referred to as $\mathrm{KC} 05$.

\section{a. $R e-X$ relation}

Understanding the influence of turbulence on the drag coefficient is explored through calculations of the $\mathrm{Re}-X$ relation. Figure 1 shows the $\operatorname{Re}-X$ relation calculated using (2.6)-(2.8) for various cases and illustrates the effect of turbulence. The uppermost curve with open triangles describes rigid spheres or drops without turbulence, calculated using the constants from Abraham (1970) $C_{0}=0.29, \delta_{0}=9.06$. The curve calculated for ice crystals without turbulence, using $C_{0}=$ 0.6, $\delta_{0}=5.83$ recommended by Böhm $(1989,1992)$, lies lower than the curve for drops and is almost indistinguishable from the corresponding curve in Böhm (1989, 1992).

Calculations for both drops and crystals that include the turbulent corrections (3.3)-(3.8) lead to a decrease in $\operatorname{Re}_{t}(X)$, asymptotically by a factor of $\psi^{1 / 2}=1 / C_{t}^{1 / 2}=$ 1/1.265. The curve calculated for the crystals with turbulence (crosses) is very close to the corresponding Re $-X$ relation from Böhm (1992, hereafter B92; solid diamonds) and to MH05 (the last curve is not shown since it merges with $\mathrm{KC} 05$ and $\mathrm{B} 92$ curves). 
It was shown in Heymsfield (2003) and MH05 that use of the upper curve from $\mathrm{KC} 02$ with $C_{0}, \delta_{0}$ for rigid spheres or drops leads to an overestimation of $\operatorname{Re}(X)$ and then of $V_{t}$ for crystal aggregates, especially at large $D$. However, this comparison here shows that our power-law representation (2.6)-(2.8) of the $\mathrm{Re}-X$ relation, the same as in $\mathrm{KC} 02$ but with $C_{0}, \delta_{0}$ for crystals and with turbulent correction, can be used for accurate evaluation of crystal fall velocities.

\section{b. The drag coefficient and crisis of drag}

Figure 2 shows calculations of the drag coefficient $C_{D}$ for rigid spheres and crystals using (2.4a) and $\operatorname{Re}(X)$ from (2.6) to (2.8), with turbulent corrections (3.1)(3.7) and without the corrections. The results are compared to the calculations of Böhm (1992), showing good agreement. At small Re, the drag coefficient $C_{D}$ decreases with increasing $\mathrm{Re}$ as $24 / \mathrm{Re}$ (Stokes law). At Re $>10-50$, this decrease in $C_{D}$ continues but more slowly leading to an asymptotic value for rigid spheres $C_{D}=$ $C_{0}=0.29$ without turbulent correction (crosses, the curves $\mathrm{B} 92$ and $\mathrm{KC} 02$ are indistinguishable). However, this behavior diverges with the experimental data (Landau and Lifshitz 1986), which show a minimum of $C_{D}$ at $\operatorname{Re} \sim 5 \times 10^{3}$, then $C_{D}$ increases to $\sim 0.5$ and then becomes almost constant till $\operatorname{Re} \sim 2 \times 10^{5}$.

These features are explained by the complex structure of the boundary layer. At $\operatorname{Re} \geq 10^{3}$, the boundary layer surrounding the falling particle is becoming turbulent and can be divided into three parts: the laminar boundary layer in the front part of the sphere, then the turbulent boundary layer farther downstream, and finally, the turbulent wake after the separation line. It was noted by Beard (1980) that the relative maximum $C_{D} \approx 0.5$ between $\operatorname{Re}=10^{4}$ and $10^{5}$ occurs because the separation line moves upstream, but it is not described by the Abraham's theory, which assumes a constant separation point. As Fig. 2 shows, the description is improved with turbulent correction: $C_{D}$ increases at Re $>2 \times 10^{3}$ to a larger value of $C_{D}=0.29 \times 1.6=0.47$ (KC05, solid spheres) and merges with the experimental data. Thus, accounting for the turbulent corrections allows describing this shift of separation line upstream at $\operatorname{Re} \geq 10^{3}$, when the boundary layer becomes wider increasing the drag.

An important and interesting feature of $C_{D}$ for rigid spheres is an abrupt decrease at $\operatorname{Re}_{\text {cris }} \sim 2 \times 10^{5}$ by a factor of 4 to 5, which is referred to as the crisis of drag (Landau and Lifshitz 1986). At $\operatorname{Re}_{\text {cris }} \sim 2$ to $3 \times 10^{5}$, the separation line moves backward, and the turbulent wake behind the body abruptly becomes much narrower, causing the drag coefficient to decrease by a

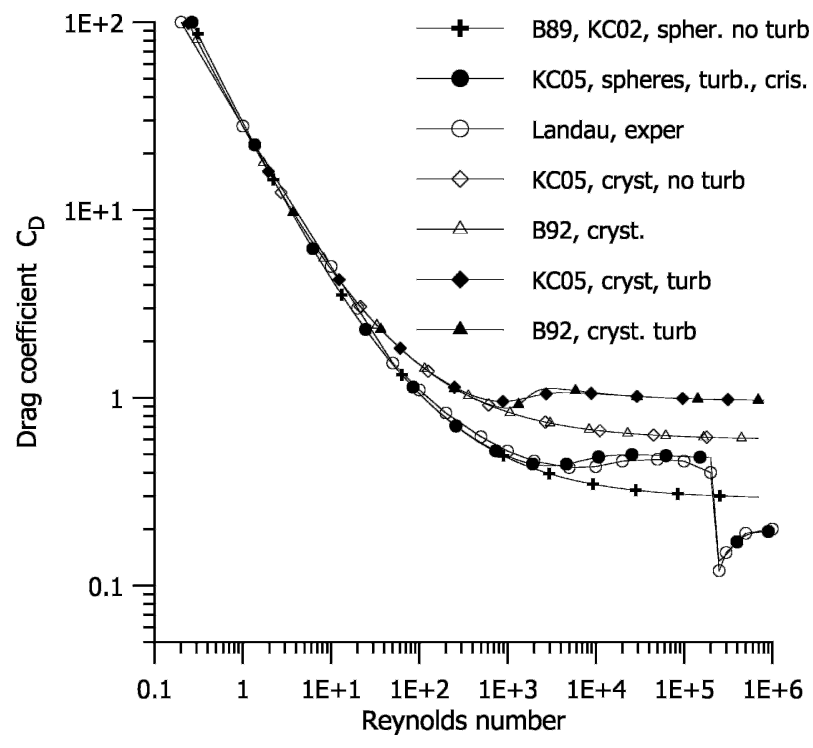

FIG. 2. Drag coefficient $C_{D}$ vs Reynolds number calculated for the spheres and crystals by various methods. Rigid spheres, $\mathrm{KC} 02$ equations, and Böhm (1989) for spheres (B89, KC02, crosses; the curves $\mathrm{B} 89$ and $\mathrm{KC} 02$ are indistinguishable); equations from this work with turbulent correction and correction for drag crisis (KC05, solid spheres); experimental data for rigid spheres from Landau and Lifshitz (1986) (Landau, open circles); equations from this work with parameters for crystals without turbulence correction (KC05, open diamonds), Bohm's equations without turbulence (B92, open triangles), this work for crystals and turbulent correction ( $\mathrm{KC} 05$, crystal, turbulence, solid diamonds), and the same case by Bohm's equations (B92, solid triangles).

factor of 4 to 5 (see Fig. 2). At $R e>R_{\text {cris }}$, the separation line begins to move upstream again, the boundary layer becomes thicker and the drag increases. We find that a continuous parameterization of this experimental data on $C_{D}$ for rigid spheres up to $\operatorname{Re}>10^{6}$ with account for turbulence and the drag crisis can be formulated as follows: (i) for $\mathrm{Re}<\mathrm{Re}_{\text {cris }} \sim 2 \times 10^{5}, C_{D}$ is described by (2.4a) with $\operatorname{Re}(X)$ from (2.6) to (2.8), $C_{0}$ and $\delta_{0}$ for the spheres and turbulent corrections (3.1)(3.7); (ii) for $\mathrm{Re} \geq \mathrm{Re}_{\text {cris }}, C_{D}$ is described using these same equations, but with a stepwise decrease of $C_{0}$ from 0.29 to 0.12 near $\operatorname{Re}_{\text {cris }}\left(X_{\text {cris }}\right)$.

Figure 2 shows satisfactory agreement between the values of $C_{D}$ calculated with these equations using the values of parameters $k=2, X_{\text {cris }}=4.8 \times 10^{10}, C_{t}=1.6$ (curve $\mathrm{KC05}$, solid circles) and the experimental data from Landau and Lifshitz (open circles) over the entire region of $\mathrm{Re}$ up to $10^{6}$. The asymptotic value at $\mathrm{Re}$ $\sim 10^{6}$ is $C_{D}=0.12 \times 1.6=0.19$, close to the value given in Landau and Lifshitz (1986).

Calculations with the same equations but with $C_{0}, \delta_{0}$ for the crystals and $k=1$ shown in Fig. 2 yield greater values of $C_{D}$ than that for the drops (KC05, open dia- 

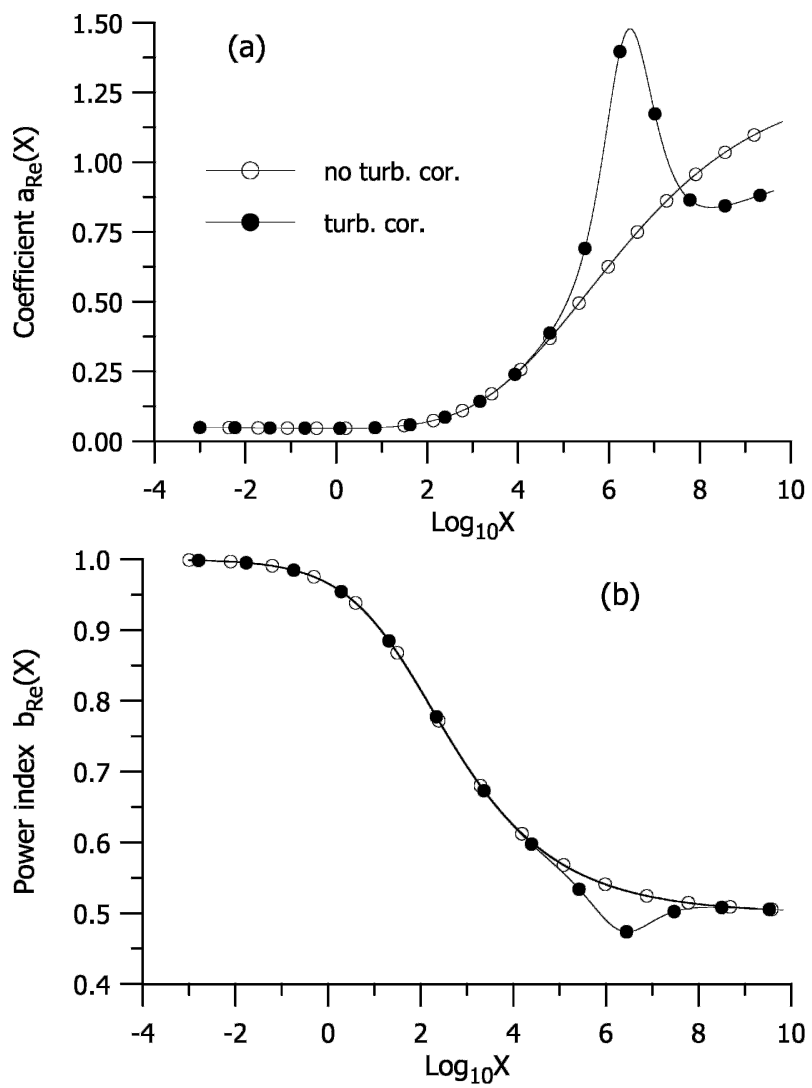

FIG. 3. (a) Coefficient $a_{\mathrm{Re}}$ and (b) power index $b_{\mathrm{Re}}$ as functions of the Best parameter $X$ with (solid circles) and without (open circles) turbulent corrections.

monds). The same increase at $\operatorname{Re}>10^{3}$ with account for the turbulent correction (solid diamonds) leads to a displacement of separation line forward, and $C_{D}$ is again in a good agreement with Böhm (1992) without and with turbulence (open and solid triangles), and with the field data for graupel from Heymsfield and Kajikawa (1987) [the last data not shown here but were in a good agreement with Böhm's (1992) calculations]. It is not clear whether the drag crisis regime can be achieved for ice crystals falling in the atmosphere. For the largest hailstones with $D \sim 10 \mathrm{~cm}$ and $V_{t} \sim 40 \mathrm{~m} \mathrm{~s}^{-1}$ (Matson and Huggins 1980; PK97), Re $\sim 3 \times 10^{5}$, which exceeds $\mathrm{Re}_{\text {cris }}$ for drops. If the drag crisis occurs for crystals near $\mathrm{Re}_{\text {cris }} \sim 2 \times 10^{5}$ as for spheres, this may mean an abrupt increase in $V_{t}$ for large hailstones at $D$ $\sim 10 \mathrm{~cm}$; however if $\mathrm{Re}_{\text {cris }}$ for the crystals is greater, the drag crisis may not occur for these sizes. This possible occurrence should be verified experimentally. Note that the drag crisis was encountered at $\mathrm{Re}_{\text {cris }} \sim 3.2 \times 10^{5}$ in experiments using dropsondes with parachutes $(\mathrm{H}$. Cole 2004, personal communication), for which this power-law formulation can be also used.

\section{c. Turbulent corrections and application to aggregates}

The turbulent corrections are especially important for crystal habits that may reach sizes $D>1 \mathrm{~cm}$, such as aggregates and hailstones. The coefficient $a_{\mathrm{Re}}(X)$ and power index $b_{\operatorname{Re}}(X)$ calculated with turbulent corrections are shown in Figs. 3a,b as universal functions (independent of crystal type) of $X$. The values $C_{0}=0.6$, $\delta_{0}=5.83$ were used as for crystals in Böhm (1992), M96, Heymsfield (2003), and MH05. Although $C_{t}=1.3$ was used in M96 to match observations from Heymsfield and Kajikawa (1987), we found that the value $C_{t}=$ 1.6 recommended by Böhm (1992; based on observations of $60 \%$ increase in $C_{D}$ by turbulence) yields better agreement for all considered crystal types. The coefficients $a_{\mathrm{Re}, t}(X)$ and $b_{\mathrm{Re}, t}(X)$ with turbulent corrections exhibit a maximum and minimum respectively in the $X$ range $10^{5}-10^{8}$ as compared to their smooth behavior without turbulent corrections. This is caused by the functions $\Delta b_{\mathrm{Re}, t}(D)$ and $\xi_{t}(D)$ defined by (3.5) and (3.7) that are shown in Figs. 4a,b for the three crystal habits as the functions of $D$. The $m-D$ and $A-D$ parameters $\alpha$, $\beta, \gamma, \sigma$ in calculations were taken from M96 and MH05 for aggregates of thin plates, from M96 for hail, and
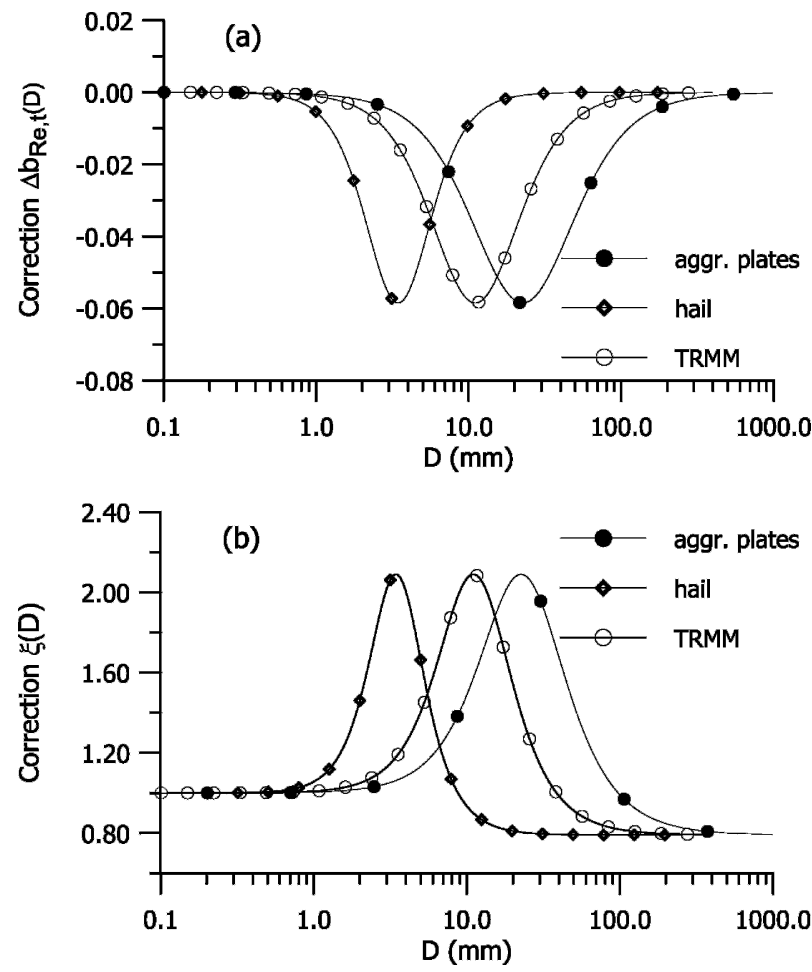

FIG. 4. (a) Corrections $\Delta b_{\mathrm{Re}, t}$ to $b_{\mathrm{Re}}$ and (b) $\xi$ to $a_{\mathrm{Re}}$ as a function of $D$ for aggregates of thin plates (solid circles), hail (diamonds), and crystal aggregates observed in tropical cirrus during TRMM described in Heymsfield (2003; open circles). 

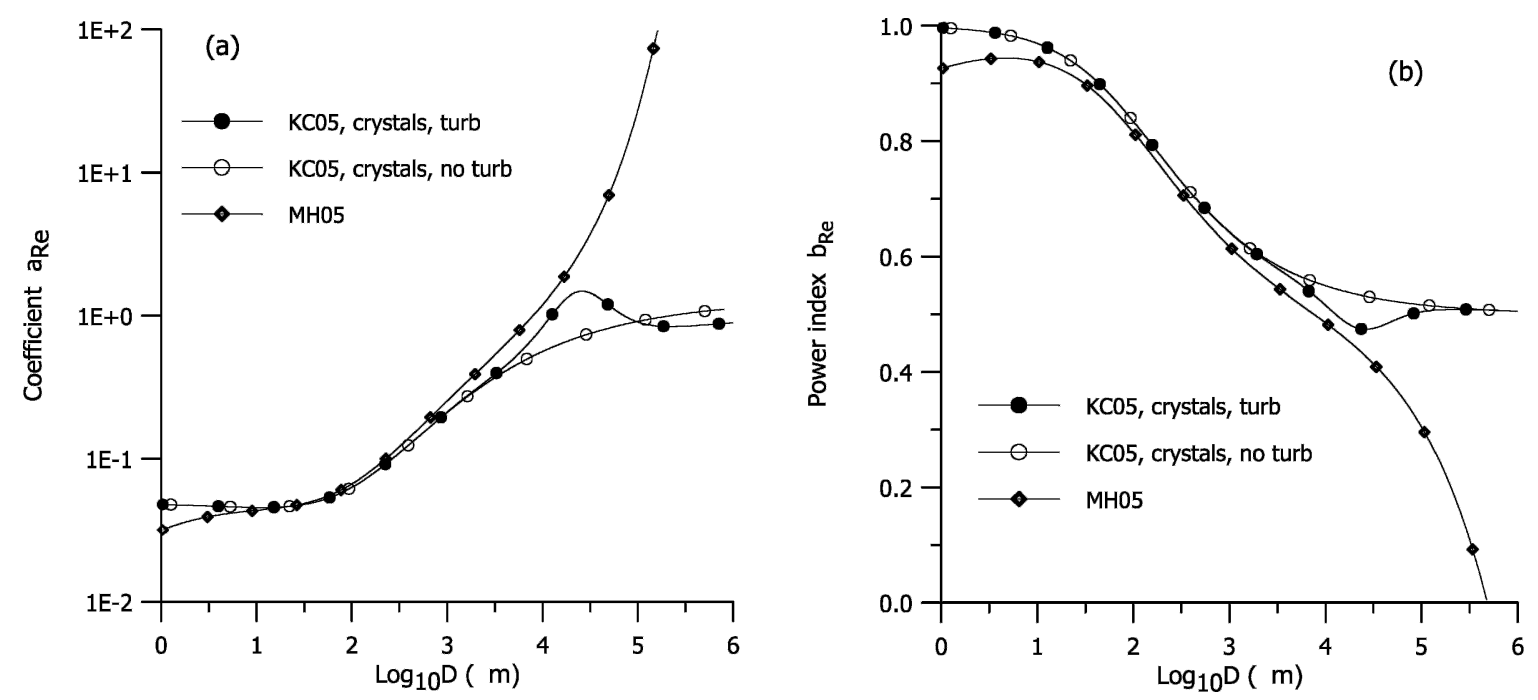

FIG. 5. (a) Coefficient of Re- $X$ power-law relation (2.6)-(2.8) $a_{\mathrm{Re}}$; (b) power index $b_{\mathrm{Re}}$ calculated for aggregates of thin plates as functions of $D$ with turbulent correction (KC05, solid circles), without correction (open circles), and compared to MH05 (diamonds).

from Heymsfield (2003) for crystal aggregates observed in tropical cirrus in the field campaign during Tropical Rain Measuring Mission (TRMM) near Kwajalein in 1999.

Although these turbulent corrections are universal functions of $X$, they differ as functions of $D$ because of different crystal habits (parameters $\alpha-\sigma$ ). Figure 4a shows that the correction $\Delta b_{\mathrm{Re}, t}$ calculated with (3.5) has a minimum of -0.06 for all three crystal habits, centered at $D=3 \mathrm{~mm}$ for hail, $1 \mathrm{~cm}$ for TRMM crystals, and $2 \mathrm{~cm}$ for aggregates of thin plates. The location of the minimum is determined by the $X-D$ relation and $m-D, A-D$ relations: the denser a crystal, the smaller $D$ of minimum $\Delta b_{\mathrm{Re}, t}$, as seen from the definition of $X$ (2.4b). Asymptotically, $\Delta b_{\mathrm{Re}, t}$ tends to zero at small and large $D$, that is, turbulence does not effect asymptotic values of the power index $b_{\mathrm{Re}}$. The correction $\xi_{t}(D)$ begins with 1 at small $D$, reaches a maximum of 2.1 at the same locations for these habits, and tends to the asymptotic value $\psi^{1 / 2}=1 / C_{t}^{1 / 2}=0.79$ according to (3.7). This explains why $a_{\operatorname{Re}}(X)$ with turbulence tends in Fig. $3 \mathrm{a}$ to a smaller value than without turbulence.

The coefficients $a_{\mathrm{Re}}$ and $b_{\mathrm{Re}}$ are shown in Fig. 5 as functions of $D$ with and without the turbulence corrections and are compared to the corresponding calculations from MH05. Figure 5 shows that all three curves for $a_{\mathrm{Re}}$ and $b_{\mathrm{Re}}$ are close at values less than $\log _{10} D \sim$ $3.7(D \sim 0.5 \mathrm{~cm})$. At higher values, the curves $\mathrm{KC} 05$ without turbulent correction begin to diverge from the other two, while $\mathrm{KC} 05$ which account for turbulence and MH05 remain sufficiently close until $\log _{10} D \sim 4.4$ $(D \sim 2.5 \mathrm{~cm})$. In the range $D=1$ to $10 \mathrm{~cm}$, the coefficients $a_{\mathrm{Re}}$ and $b_{\mathrm{Re}}$ in KC05 with turbulence have, re- spectively, local maximum of $1.48(\sim 50 \%$ greater than the asymptotic) and minimum of 0.47 (smaller than the asymptotic), both centered at $D \sim 2.5 \mathrm{~cm}$. This is caused by the behavior of corrections $\xi_{t}$ and $\Delta b_{\mathrm{Re}, t}$ shown in Fig. 4. At values greater than $\log _{10} D \sim 5$ ( $D$ $=10 \mathrm{~cm}$ ), coefficients $a_{\mathrm{Re}}$ and $b_{\mathrm{Re}}$ in $\mathrm{KC} 05$ scheme tend to their asymptotic values with turbulence 1.02 and 0.5 defined by (4.5), and coefficients in MH05 scheme tend to the larger and smaller values, respectively. Since the aggregates larger than $\sim 5 \mathrm{~cm}$ are very rare in the atmosphere, the coefficients $a_{\mathrm{Re}}$ and $b_{\mathrm{Re}}$ in both schemes MH05 and KC05 are sufficiently close.

These variations of $a_{\mathrm{Re}}$ and $b_{\mathrm{Re}}$ determine the behavior of the coefficients $A_{v}, B_{v}$ of the velocity power law shown in Figs. 6a,b. Coefficients calculated with both schemes, $\mathrm{KC} 05$ and $\mathrm{MH} 05$, are close in the range of $\log _{10} D(\mu \mathrm{m})$ from 2 to 4.5 , then begin to diverge. However, the fall speeds (Fig. 6c) in both schemes are very close up to $\log _{10} D=5(D=10 \mathrm{~cm})$. The difference in $V_{t}$ does exceed $4.2 \%$ up to $D=5 \mathrm{~cm}$. Aggregates of these sizes were observed in frontal systems near $0^{\circ} \mathrm{C}$ (Lawson et al. 1998). Calculated fall speeds are close in Fig. 6c to the field data on $V_{t}$ by Locatelli and Hobbs (1974) for aggregates of unrimed radiating assemblages and aggregates of unrimed side planes.

An interesting feature of both $\mathrm{KC} 05$ and $\mathrm{MH} 05$ schemes is that they predict a decrease in $V_{t}$ at $D \geq 1$ $\mathrm{cm}$. It is reached by subtracting an additional term from the Böhm's $\mathrm{Re}-X$ relation in the MH05 scheme [see (2.15)], and by introducing the turbulent correction in KC05 scheme. We used the values $\beta=1.8, \sigma=1.88$ from Table 1 in M96 for these aggregates. If the asymptotic value is used without turbulent correction $b_{\mathrm{Re}}=0.5$, 

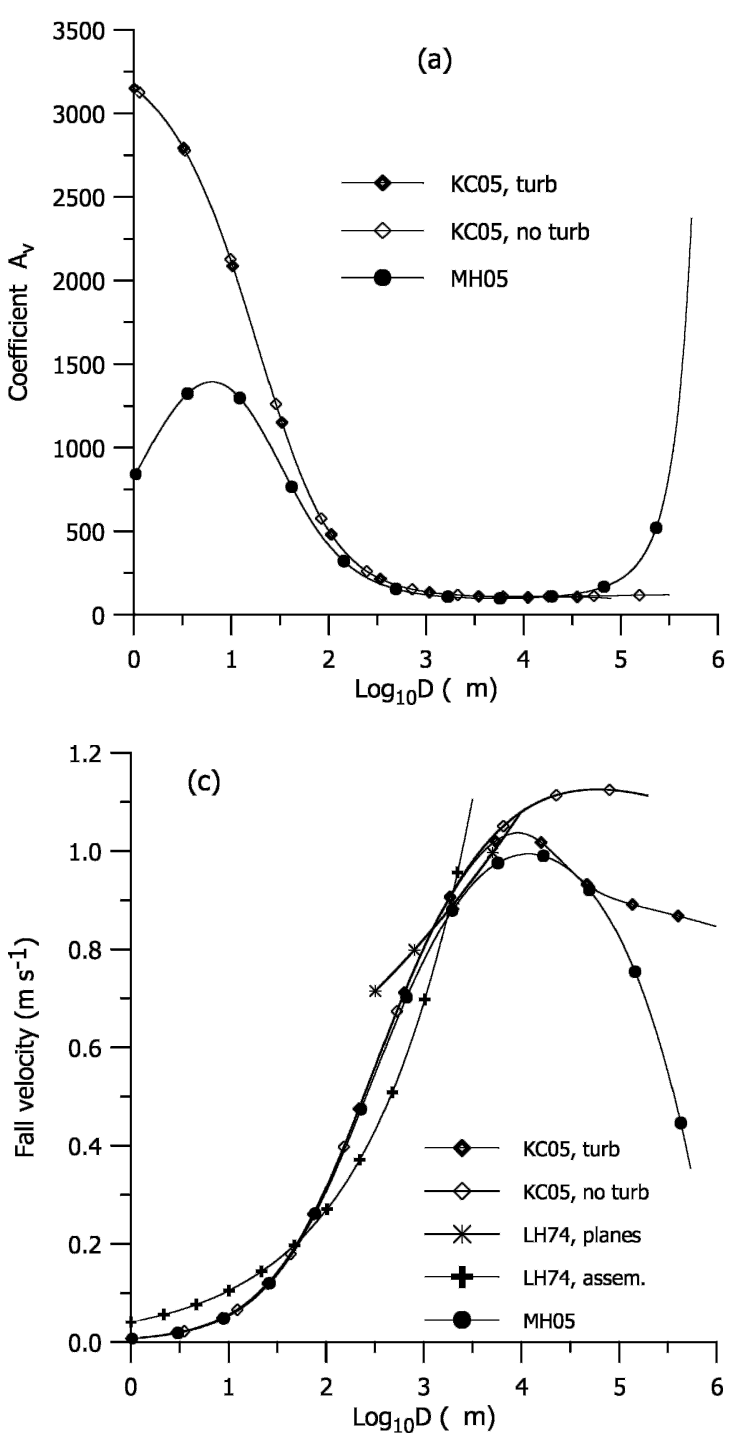

then (2.14) gives $B_{v}=0.5 \times(1.8-1.88+2)-1=$ -0.04 , predicting a decrease in $V_{t}$ with increasing $D$. However, this asymptotic and negative value of $B_{v}$ are attained at unrealistically large $D \sim 100 \mathrm{~cm}$ (Figs. 5b, $6 \mathrm{~b}$ ), and $\mathrm{KC} 02$ scheme without turbulence does not predict such inverse behavior of $V_{t}(D)$. The situation is changed with account for turbulent correction: due to negative $\Delta b_{\mathrm{Re}}$ (see Fig. $4 \mathrm{a}$ ), $B_{v}$ becomes negative at $D$ $\sim 1$ to $10 \mathrm{~cm}$, and $V_{t}$ begins to decrease with $D$ in this region. A minimum $b_{\mathrm{Re}}=0.47$ is reached much earlier, at $D=2.5 \mathrm{~cm}$. Evaluation again from (2.14) with these $\beta, \sigma$ gives $B_{v}=-0.1$ (Fig. $6 \mathrm{~b}$ ); that is, the fall speed decreases with the maximum rate as $V_{t} \sim D^{-0.1}$ near this point. This decrease continues, although somewhat slower, at larger $D$ (Fig. 6c).

MH05 pointed out that this decrease of fall speeds

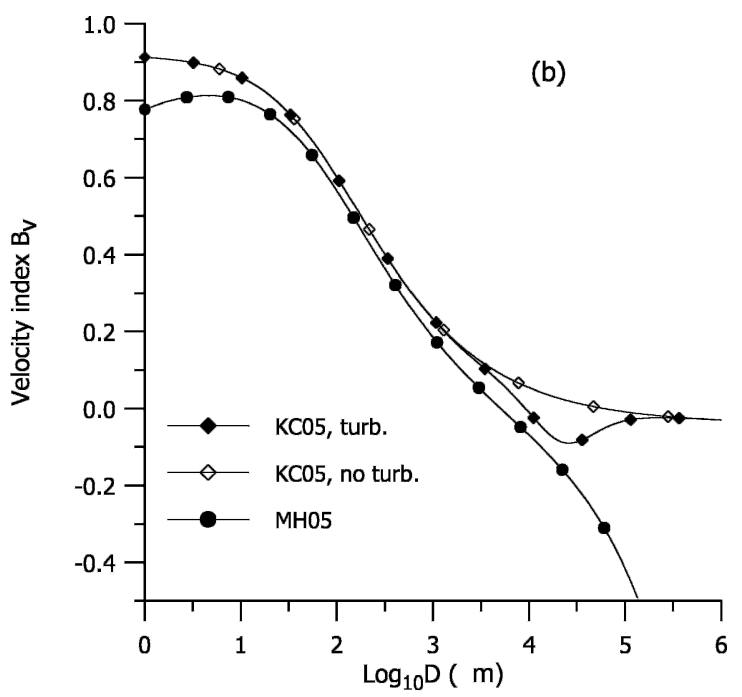

Fig. 6. (a) Coefficient $A_{v}$, (b) power-law index $B_{v}$, and (c) fall velocity $V_{t}$ for unrimed aggregates of thin plates calculated with Eqs. (2.13)-(2.15) of this work, $C_{0}, \delta_{0}$ for crystals, with turbulent corrections (KC05, solid diamonds), and without turbulent correction (KC05, open diamonds), compared to $A_{v}, B_{v}$ calculated in MH05 with modified equations of $\mathrm{KC} 02$ (solid circles) and to the field data on $V_{t}$ by Locatelli and Hobbs (1974) for aggregates of unrimed radiating assemblages (crosses) and aggregates of unrimed side planes (asterisks).

with increasing aggregates size would lead to a suppressed gravitational aggregation, and noted that the KC02 scheme with $C_{0}, \delta_{0}$ for the drops does not predict this effect. However, as shown in Fig. 6c, the current scheme yields $V_{t}$ very close to the MH05 scheme (especially designed for aggregates) and predicts a decrease in fall speeds for the larger aggregates and suppression of further aggregation.

Another comparison of the MH05 and $\mathrm{KC05}$ schemes was performed for aggregates observed in tropical cirrus during TRMM campaign (Heymsfield 2003) using the same values of the parameters from Heymsfield (2003) as in Fig. 4. The $V_{t}$ shown in Fig. 7 were calculated with $\mathrm{KC} 05$ scheme for the conditions of experiment, and recalculated at pressure $p=1000 \mathrm{hPa}$, using $C_{p T}$ correction (5.3c), and compared to the cor- 
responding data in Heymsfield (2003), where calculations were performed with MH05 scheme. Figure 7 exhibits good agreement between the two schemes. The curve calculated with $\mathrm{KC} 02$ scheme using the same $\alpha, \beta$, $\gamma, \sigma$ but $C_{0}, \delta_{0}$ for the drops (crosses) is shown for comparison. However, as shown in Fig. 7, the current scheme yields the same results for aggregates as the MH05 scheme, the difference not exceeding 5\%. The fall speeds at these two heights are used in section $6 \mathrm{f}$ for verification of the altitude correction (5.3c).

\section{d. Other crystal habits}

A comparison of the $\mathrm{KC} 02-\mathrm{KC} 05$ scheme with the other schemes and experimental data for various crystal habits is shown in Figs. 8-10. All calculations were performed with $C_{0}, \delta_{0}$ for crystals and $\alpha-\sigma$ parameters were taken from Table 1 in M96. Note that for crystal habits shown in Figs. 8-10, $\mathrm{Re}<10^{3}$ and the turbulent corrections are insignificant, so that actually the $\mathrm{KC} 02$ scheme is tested with $C_{0}, \delta_{0}$ for crystals. A very good agreement of the $\mathrm{KC} 02-\mathrm{KC} 05$ scheme with M96 calculations and experimental data from Heymsfield and Kajikawa (1987) is seen for hexagonal plates (Fig. 8), with M96 calculations and field data from Locatelli and Hobbs (1974) for rimed columns and dendrites (Fig. 9), with M96 calculations and experimental data from Heymsfield (1972) for hexagonal columns (Fig. 10), and with experimental parameterization from Brown (1970) and calculations from MH05 for plates with sector

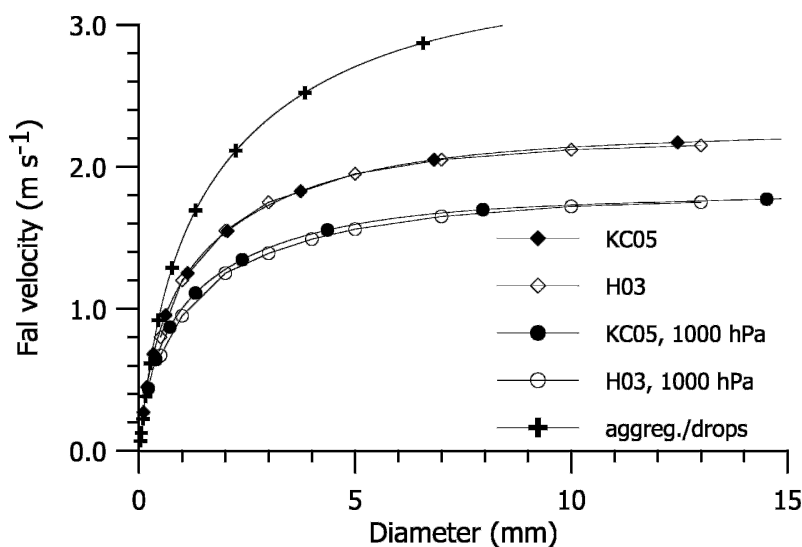

FIG. 7. Fall velocities calculated with equations of this work (KC05) and crystal parameters $(\alpha, \beta, \gamma, \sigma)$ from TRMM data by Heymsfield (2003) for the conditions of experiment (solid diamonds) and for $p=1000 \mathrm{hPa}$ (solid circles), compared to the calculations from Heymsfield (2003), labeled H03 (open diamonds and circles). The difference does not exceed $5 \%$ at 1000 $\mathrm{hPa}$. The curve calculated with $\mathrm{KC} 02$ scheme using the same $\alpha, \beta$, $\gamma, \sigma$ but $C_{0}, \delta_{0}$ for the drops (crosses, aggregates/drops) and cited by Heymsfield (2003) in Fig. 4 is given for comparison.

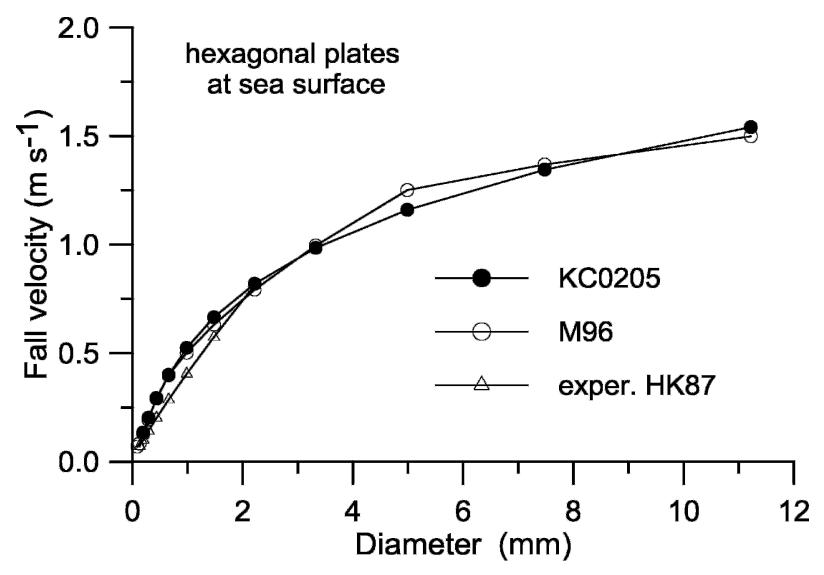

FIG. 8. Fall velocities of hexagonal plates calculated with equations of $\mathrm{KC} 02$ or this work and $C_{0}, \delta_{0}$ parameters for crystals (KC02-KC05, solid circles) and by Mitchell (1996; open circles) compared up to $12 \mathrm{~mm}$, and parameterization by Heymsfield and Kajikawa (1987) based on experimental data and extended to 2.2 $\mathrm{mm}$ (open triangles).

branches (Fig. 10). Results for the other dendritic crystals (plates with broad branches, stellars) are close to those for sector branches and to those calculated in MH05 for these crystal types. In accord with conclusions from $\mathrm{MH} 05$, the agreement improved when incorporating new values of $C_{0}, \delta_{0}$ in the $\mathrm{KC} 02$ scheme for the crystals.

This scheme could be applied for various snowflakes, but it meets the problem of high variability of their properties, in particular, habits and densities. This leads sometimes to contradictive results. As an example, Magono and Nakamura (1965) predicted decrease of

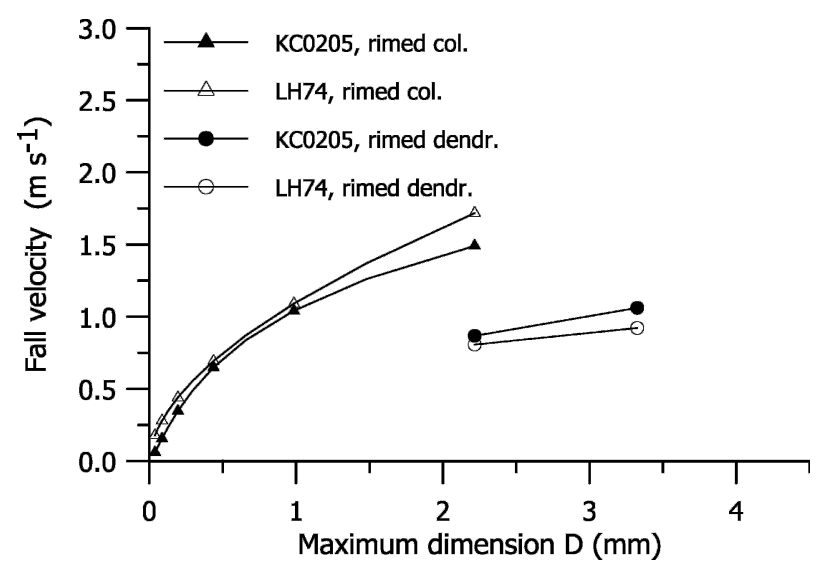

FIG. 9. Fall velocities for densely rimed columns and dendrites calculated with equations of $\mathrm{KC} 02$ or this work and $C_{0}, \delta_{0}$ parameters for crystals ( $\mathrm{KC} 02-\mathrm{KC} 05$, solid triangles and circles) compared to the field data by Locatelli and Hobbs (1974; open triangles and circles). 


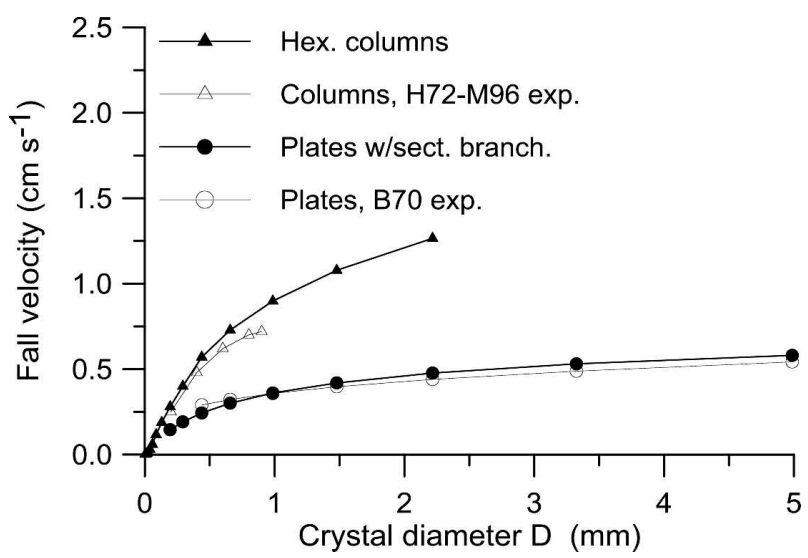

FIG. 10. Fall velocities for hexagonal columns (solid triangles) and plates with sector branches (solid circles) calculated with equations of this work and compared to experimental data for columns from Heymsfield (1972) adapted in Mitchell (1996) (H72-M96, open triangles) and parameterization of Brown 1970 (B70) for plates cited in M96 and MH05 (open circles).

snowflakes fall velocity as $D^{-1 / 2}$ explained by the authors by a significant density decrease with growing size. In contrast, Jiusto and Bosworth (1971) observed and parameterized velocity increase with size as $D^{0.2}$. This uncertainty in snowflakes properties and their high variability indicates a necessity of their further studies, in particular, parameterization of the $A-D, m-D$, and density- $D$ relations.

\section{e. Hail}

The final test of this scheme was performed with application to hail. This crystal habit achieves the greatest sizes, $D \sim 10 \mathrm{~cm}$, numbers $\operatorname{Re} \sim 3 \times 10^{5}, X \sim 10^{11}$, and is especially sensitive to the turbulent corrections. Figure 11 shows that calculations with the KC05 scheme and $\alpha-\sigma$ parameters from M96 are again in very close agreement with the experimental data for hailstones from Matson and Huggins (1980, hereafter MH80), and to M96 and Böhm (1992) who also used these experimental data for comparison. The difference between MH80 and KC05 with turbulence correction is less than $3.5 \%$ at $D=50 \mathrm{~mm}$ and $2 \%$ at $D=100 \mathrm{~mm}$. Without turbulent correction, $V_{t}$ is $26 \%$ higher at $D=100 \mathrm{~mm}$, which is explained by the effect of turbulence described by (4.4): accounting for turbulence leads to a reduction in $a_{\mathrm{Re}}$ and thereby in $V_{t}$ by a factor of $1 / C_{t}^{1 / 2}=1 / 1.26$. Figure 11 shows that our choice of $C_{t}=1.6$ recommended by Böhm (1992) leads to agreement with the MH80 data. Note that the correction $\xi(D)$ for hail lies in the region of small $D \sim 2-5 \mathrm{~mm}$ (see Fig. 4) and does not lead to a noticeable effect in fall speeds at large sizes in contrast to the aggregates (cf. with Fig. 6c).

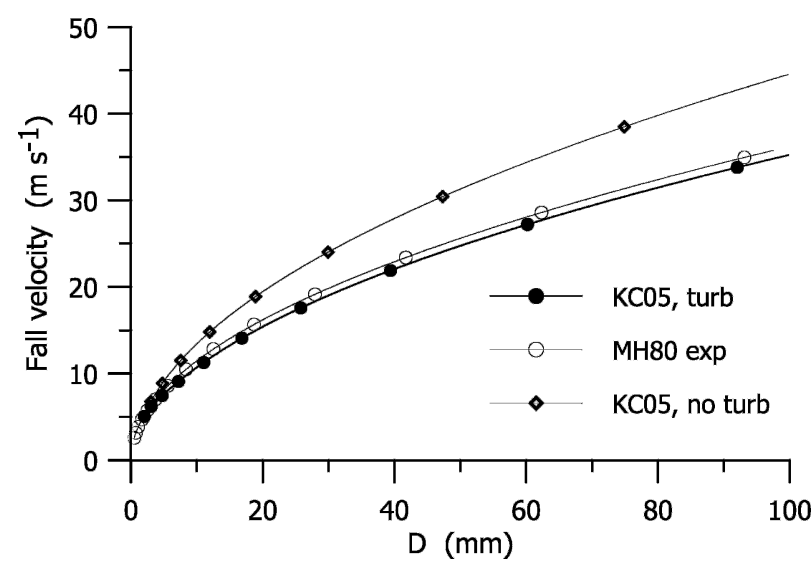

FIG. 11. Terminal velocity of hailstones $V_{t}$ calculated with equations from this work, $\delta_{0}$ and $C_{0}$ for crystals, and $C_{t}=1.6$ with turbulent correction ( $\mathrm{KC} 05$, solid circles) and without correction (diamonds) compared to the experimental data from MH80 (open circles). The difference between KC05 with turbulence and MH80 is less than $3.5 \%$ at $D=50 \mathrm{~mm}$ and $2 \%$ at $D=100 \mathrm{~mm} ; V_{t}$ without turbulent correction is higher by $26 \%$ at $D=100 \mathrm{~mm}$.

\section{f. Calculations of the altitude correction}

The altitude or pressure-temperature correction (5.3c) was verified by comparison with results from Beard (1980), who fitted slopes of the measured or calculated $C_{D}(\mathrm{Re})$ curves or from the similarity arguments, and then parameterized by constructing an empirical interpolation function $C_{p T}(\mathrm{Re})$ linear by $\ln (\mathrm{Re})$ between the viscous $C_{p T 0}$ and aerodynamic $C_{p T \infty}$ limits same as (5.4), (5.5), but with the stepwise constraints $C_{p T}=C_{p T 0}$ at $\operatorname{Re}<0.2$, and $C_{p T}=C_{p T \infty}$ at $\operatorname{Re}>10^{3}$. (Note that with our method, we did it in reverse order: obtained first an analytical expression for $C_{p T}$ and then its asymptotes). We performed calculations of $C_{p T}$ for the same altitude variation as in Beard (1980) between the reference level with $p=770 \mathrm{hPa}, T_{c}=0^{\circ} \mathrm{C}$, and the top of the atmosphere with $p=232 \mathrm{hPa}, T_{c}=-55^{\circ} \mathrm{C}$. Figure 12a show that all four curves in the region $1<$ $\operatorname{Re}<5 \times 10^{2}$ lie close, the difference slightly increasing for smaller particles at $\mathrm{Re} \sim 0.1$ and around large $\mathrm{Re}$ $\sim 10^{3}$. The latter local maximum (absent on the curve without turbulence) is caused by the turbulent correction in our scheme and allows explanation of experimentally observed local increase in $C_{p T}$ near $\operatorname{Re} \sim 10^{3}$ that is not described by Beard's monotonous function. This agreement in the altitude correction indicates a validity of our scheme, which provides a theoretical justification and smoothing of Beard's parameterization.

Another test of (5.3c) for the $C_{p T}$ correction was performed by comparing its values calculated with $(5.3 \mathrm{c})$ and $b_{\mathrm{Re}, t}$ from (3.4) for crystal aggregates in tropical 

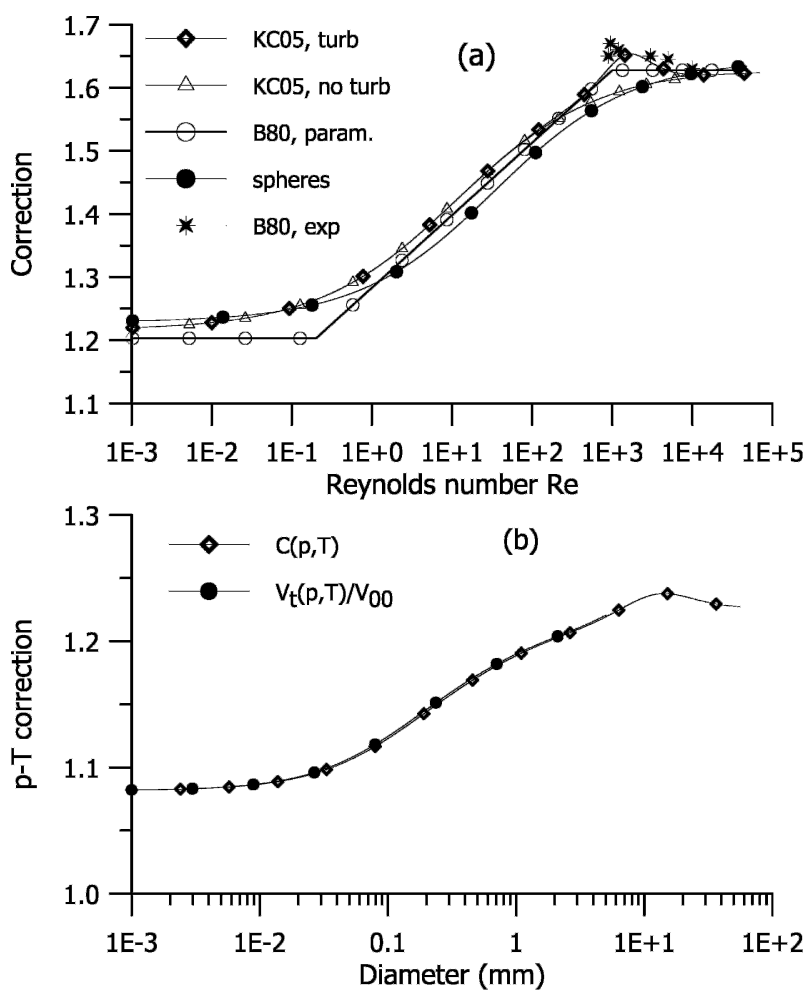

FIG. 12. The altitude (pressure-temperature) correction. (a) Calculations using (5.3c) for crystals with turbulence (KC05, solid diamonds) and without turbulence ( $\mathrm{KC} 05$, open triangles), Beard's (1980) general parameterization (open circles), calculations for spheres (solid circles) and some experimental points from Beard (1980) located higher than Beard's parameterization (asterisks); (b) correction $C(p, T)$ for tropical cirrus aggregates as in Fig. 7 calculated from (5.3c) and as the ratio $V_{t}(p, T) / V_{t}(p=$ $\left.1000, T=-5^{\circ} \mathrm{C}\right)$.

cirrus (Heymsfield 2003) with the direct calculation of the velocities ratio $C_{p T}=V_{t}(p, T) / V_{t}(p=1000, T=$ $-5^{\circ} \mathrm{C}$ ) calculated with the equations of sections 2 and 3 (see Fig. 7). Figure 12b shows that both methods give very close results over the entire $D$ range, where the $C_{p T}$ correction significantly varies with $D$ between the viscous and aerodynamic limits (5.4), (5.5).

\section{Summary and conclusions}

This paper extends our previous research (Khvorostyanov and Curry 2002) on developing a power-law representation for the terminal velocities of hydrometeors with coefficients that are continuous analytical functions over the particle size spectrum. In particular, we improve the results of the $\mathrm{KC} 02$ parameterization for larger particles (e.g., aggregates and hail), and show that the further empirical corrections to the $\mathrm{KC} 02$ scheme are not needed. The results of this paper are summarized briefly below.
1) The $\mathrm{KC} 02$ parameterization is refined (a) by using more precise parameters for the ice crystals (the drag coefficient for the potential flow and the boundary layer depth) with account for the surface roughness, and (b) by introducing turbulent corrections into the drag coefficient using Böhm's (1992) interpolation function.

2) The turbulent corrections are formulated analytically in terms of the Reynolds number, coefficients of the $\operatorname{Re}-X$ power law, and the terminal velocity power law.

3) This modification of the $\mathrm{KC} 02$ scheme is applicable for high values of $X$ and $\mathrm{Re}$, provides a smooth analytical description of both power laws up to $X>$ $10^{12}$ and $\operatorname{Re}>10^{6}$ without negative coefficients, and has correct asymptotic limits for both small and large particles, which are found analytically. In application for a particular case of snow aggregates, where $X$ does not exceed $10^{7}$ for the largest particles, our new scheme yields the results very close to the parameterization by $\mathrm{MH} 05$ and confirms it validity in the entire size range of the aggregates.

4) In the large particle limit, the turbulent correction leads to a decrease of the terminal velocity by the factor $1 / C_{t}^{1 / 2}$, or by $\sim 30 \%$ with $C_{t}=1.6$ recommended by Böhm (1992), which removes the tendency of the $\mathrm{KC} 02$ scheme, noted by MH05, to overestimate fall speeds by up to $30 \%$ for large aggregates.

5) The new scheme predicts that besides the limits for small and large sizes, there is an intermediate region of $X \sim 10^{6}$, where the coefficients of the $\operatorname{Re}-X$ power law have local minima and maxima. This leads to the local minimum and negative values in the velocity power law index for aggregates, and causes a decrease in terminal velocity with increasing aggregate size in the region $D \sim 1-10 \mathrm{~cm}$, hampering further aggregation. This feature is similar to that predicted by the MH05 scheme, but results here from the turbulent corrections.

6) An analytical correction is derived for the altitude (or temperature and pressure) dependence of terminal velocities, which is size-dependent and converts into correct viscous and aerodynamic limits for small and large particles, respectively. The derivation here is different from that in Beard (1980), but provides similar (and smoother) results. The altitude correction predicts higher velocity increase with altitude for the large particles and increase in velocity difference between large and small particles with increasing height, and implies acceleration of coagulation and accretion growth of cloud particles. 
7) The results of the new scheme are verified by comparison with experimental data and other theoretical schemes. The accuracy of our modified scheme is found to be sufficiently high, the difference with the experimental data is generally within a few percent.

The scheme uses nine parameters of three types: (a) constants for the boundary layer, $C_{0}, \delta_{0}$ (different for drops and crystals); (b) parameters of the interpolation function between laminar and turbulent regime, $C_{t}, k$, and $X_{0}$; and (c) parameters of the $m-D$ and $A-D$ relations, $\alpha, \beta, \gamma, \sigma$. The parameters of types (a) and (b) are known and predetermined from aerodynamic experiments. Parameters of type (c) are based on the physical properties of the particles, obtained from Table 1 in Mitchell (1996), and from Heymsfield (2003) for aggregates in tropical cirrus.

Thus, our scheme is theoretically based and does not contain any parameters that are tuned to observed terminal velocities. The major skill in its applications for any crystal habit is in the right choice of the $\alpha-\sigma$ parameters, which are based on the parameterization of the crystal physical properties. These parameters are available for many crystal habits as cited in the text; however, they are often contradictory among different sources and further studies are needed for their refinement and generalization, such as in Mitchell (1996). Unfortunately, data on the height- or temperature- and pressure-dependence of the fall speeds of various crystal habits are still scarce, and their collection is needed to verify the theoretical parameterizations.

The scheme described here can be applied directly in cloud models with bin-resolved microphysics, and can be used for development of fall speed parameterization averaged over the size spectra of various species in the bulk microphysical models and GCMs. An example of such a parameterization was given in $\mathrm{KC} 02$ for rain in application to the Colorado State University (CSU) GCM (Fowler et al. 1996). Another applications may include remote sensing similar to Sassen (1980), Matrosov and Heymsfield (2000) or analytical parameterizations of the drop and crystal size spectra where parameters of the gamma distributions directly contain parameters of the fall speed power law (e.g., Mitchell 1988, 1994; Khvorostyanov and Curry 1999a,b).

Acknowledgments. This research was supported by grants from the Department of Energy Atmospheric Radiation Measurement Program. We thank Dave Mitchell and Andy Heymsfield for the useful discussions that stimulated this work, the two anonymous reviewers for their comments and remarks that improved the manuscript, and Jody Norman for help in preparing the manuscript.

\section{APPENDIX}

\section{List of Symbols}

$A$

$A_{v}$

$a_{\mathrm{Re}}$

$b_{\mathrm{Re}}$

$B_{v} \quad$ Power index in the velocity power law

$C_{0} \quad$ Drag coefficient for potential flow

$C_{1}=4 /\left(\delta_{0}^{2} C_{0}^{1 / 2}\right)$ Constant in equations

$C_{D} \quad$ Drag coefficient

$C_{p T} \quad$ Pressure and temperature correction to the fall velocity

$C_{t} \quad$ Asymptotic value of the turbulent correction to $C_{D}$ in (3.1), (3.2)

$D \quad$ Particle diameter or maximum length

$D_{\text {sc }} \quad$ Scaling diameter that separates potential and aerodynamic flows

$F_{D} \quad$ Drag force

$F_{b} \quad$ Buoyancy force

$g \quad$ Acceleration of gravity

$k \quad$ Power index in the interpolation function $\psi(X)$ (3.2) for $C_{D}$

$\begin{array}{ll}m & \text { Mass of a drop or a crystal } \\ m g & \text { Gravitational force }\end{array}$

$\mathrm{Re} \quad$ Reynolds number

$\mathrm{Re}_{\text {cris }} \quad$ Reynolds number for the onset of drag crisis for rigid spheres

Droplet or crystal radius

Terminal velocity

Body volume

Best (or Davies) number

Scaling Best parameter in the turbulence interpolation function (3.2)

Best number for the onset of drag crisis for rigid spheres

Scaling Best number that separates potential and aerodynamic flows

Coefficient in mass-dimension relation

Power index in mass-dimension relation

Coefficient in area-dimension relation

Boundary layer depth

Coefficient in the similarity relation for the boundary layer depth $\delta$

Fluid (air) dynamic viscosity

Fluid (air) kinematic viscosity

$\nu=\eta / \rho_{F}$

$\rho_{a}, \rho_{i}, \rho_{w}$,

$\rho_{b}, \rho_{F}$,

$\sigma$
Densities of air, ice, water

Densities of the body and fluid

Power index in area-dimension relation 


\section{REFERENCES}

Abraham, F., 1970: Functional dependence of drag coefficient of a sphere on Reynolds number. Phys. Fluids, 13, 2194-2195.

Beard, K. V., 1980: The effect of altitude and electrical force on the terminal velocity of hydrometeors. J. Atmos. Sci., 37, 1363-1374.

Böhm, J. P., 1989: A general equation for the terminal fall speed of solid hydrometeors. J. Atmos. Sci., 46, 2419-2427.

— 1992: A general hydrodynamic theory for mixed-phase microphysics. Part I: Drag and fall speeds of hydrometeors. Atmos. Res., 27, 253-274.

Brown, S. R., 1970: Terminal velocities of ice crystals. M.S. thesis, Dept. of Atmospheric Sciences, Colorado State University, $52 \mathrm{pp}$.

Cotton, W. R., and R. A. Anthes, 1989: Storm and Cloud Dynamics. Academic Press, 883 pp.

Fowler, L. D., D. A. Randall, and S. A. Rutledge, 1996: Liquid and ice cloud microphysics in the CSU general circulation model. Part I: Model description and simulated microphysical processes. J. Climate, 9, 489-529.

$\mathrm{Fu}$, Q., S. K. Krueger, and K. N. Liou, 1995: Interactions of radiation and convection in simulated tropical cloud clusters. $J$. Atmos. Sci., 52, 1310-1328.

Heymsfield, A. J., 1972: Ice crystals terminal velocities. J. Atmos. Sci., 29, 1348-1357.

, 2003: Properties of tropical and midlatitude ice cloud particle ensembles. Part I: Median mass diameters and terminal velocities. J. Atmos. Sci., 60, 2573-2591.

—_, and M. Kajikawa, 1987: An improved approach to calculating terminal velocities of plate-like crystals and graupel. $J$. Atmos. Sci., 44, 1088-1099.

- , and J. Iaquinta, 2000: Cirrus crystals terminal velocities. $J$. Atmos. Sci., 57, 916-938.

Jensen, E. J., O. B. Toon, D. L. Westphal, S. Kinne, and A. J. Heymsfield, 1994: Microphysical modeling of cirrus. 1. Comparison with 1986 FIRE IFO measurements. J. Geophys. Res., 99, $10421-10442$.

Jiusto, J. E., and G. E. Bosworth, 1971: Fall velocity of snowflakes. J. Appl. Meteor., 10, 1352-1354.

Kessler, E., 1969: On the Distribution and Continuity of Water Substance in Atmospheric Circulation. Meteor. Monogr., No. 10, Amer. Meteor. Soc., 84 pp.

Khvorostyanov, V. I., 1995: Mesoscale processes of cloud formation, cloud-radiation interaction and their modelling with explicit spectral microphysics. Atmos. Res., 39, 1-67.

— , and J. A. Curry, 1999a: Toward the theory of stochastic condensation in clouds. Part I: A general kinetic equation. $J$. Atmos. Sci., 56, 3985-3996.

$\longrightarrow$, and — 1999: Toward the theory of stochastic condensation in clouds. Part II: Analytical solutions of the gammadistribution type. J. Atmos. Sci., 56, 3997-4013.

- and - 2002: Terminal velocities of droplets and crystals: Power laws with continuous parameters over the size spectrum. J. Atmos. Sci., 59, 1872-1884.

— — - I. Gultepe, and K. Strawbridge, 2003: A springtime cloud over the Beaufort Sea polynya: 3D simulation with explicit spectral microphysics and comparison with observations. J. Geophys. Res., 108, 4296, doi:10.1029/2001JD001489.

Knight, N. C., and A. J. Heymsfield, 1983: Measurement and interpretation of hailstone density and terminal velocity. J. Atmos. Sci., 40, 1510-1516.
Krueger, S. K., Q. Fu, K. N. Liou, and H.-N. S. Chin, 1995: Improvement of an ice-phase microphysics parameterization for use in numerical simulation of tropical convection. J. Appl. Meteor., 34, 281-287.

Landau, L. M., and E. M. Lifshitz, 1986: Hydrodynamics (in Russian). Nauka, 733 pp.

Lawson, R. P., R. E. Stewart, and L. J. Agnus, 1998: Observations and numerical simulations of the origin and development of very large snowflakes. J. Atmos. Sci., 55, 3209-3229.

Lin, Y. L., R. D. Farley, and H. D. Orville, 1983: Bulk parameterization of the snow field in a cloud model. J. Climate Appl. Meteor., 22, 1065-1092.

Locatelli, J. D., and P. V. Hobbs, 1974: Fall speeds and masses of solid precipitation particles. J. Geophys. Res., 79, 2185-2197.

Magono, C., and T. Nakamura, 1965: Aerodynamic studies of falling snowflakes. J. Meteor. Soc. Japan, 43, 139-147.

Matrosov, S. Y., and A. J. Heymsfield, 2000: Use of Doppler radar to assess ice cloud particle fall velocity-size relations for remote sensing and climate studies. J. Geophys. Res., 105, 22 427-22 436

Matson, R. J., and A. W. Huggins, 1980: The direct measurement of the sizes, shapes and kinematics of falling hailstones. $J$. Atmos. Sci., 37, 797-816.

Mitchell, D. L., 1988: Evolution of snow-size spectra in cyclonic storms. Part I: Snow growth by vapor deposition and aggregation. J. Atmos. Sci., 45, 3431-3451.

- 1994: A model predicting the evolution of ice particle size spectra and radiative properties of cirrus clouds. Part I: Microphysics. J. Atmos. Sci., 51, 797-816.

_, 1996 : Use of mass- and area-dimensional power laws for determining precipitation particle terminal velocities. J. Atmos. Sci., 53, 1710-1723.

, and A. J. Heymsfield, 2005: Refinements in the treatment of ice particle terminal velocities: Highlighting aggregates. $J$. Atmos. Sci., 62, 1637-1644.

Morrison, H., J. A. Curry, and V. I. Khvorostyanov, 2005a: A new double-moment microphysics parameterization. Part I: Description. J. Atmos. Sci., 62, 1665-1677.

$\longrightarrow,-$ M. D. Shupe, and P. Zuidema, 2005b: A new doublemoment microphysics scheme for application in cloud and climate models. Part II: Single-column modeling of arctic clouds. J. Atmos. Sci., 62, 1678-1693.

Pruppacher, H. R., and J. D. Klett, 1997: Microphysics of Clouds and Precipitation. 2d ed. Kluwer Academic, 954 pp.

Rogers, R. R., 1976: A Short Course in Cloud Physics. A. Wheaton \& Co., $266 \mathrm{pp}$

Rutledge, S. A., and P. V. Hobbs, 1983: The mesoscale and microscale structure and organization of clouds and precipitations in midlatitude cyclones. VIII. A model for the "seederfeeder" process in warm frontal rainbands. J. Atmos. Sci., 40, $1185-1206$

Sassen, K., 1980: Remote sensing of planar ice crystal fall attitudes. J. Meteor. Soc. Japan, 58, 422-429.

Starr, D. O'C., and S. K. Cox, 1985a: Cirrus clouds. Part I: A cirrus cloud model. J. Atmos. Sci., 42, 2663-2681.

_ and $\longrightarrow$ 1985b: Cirrus clouds. Part II: Numerical experiment on the formation and maintenance of cirrus. J. Atmos. Sci., 42, 2682-2694.

Tao, W.-K., J. Simpson, and S.-T. Soong, 1991: Numerical simulation of a subtropical squall line over Taiwan Strait. Mon. Wea. Rev., 119, 305-328. 
Copyright of Journal of the Atmospheric Sciences is the property of American Meteorological Society and its content may not be copied or emailed to multiple sites or posted to a listserv without the copyright holder's express written permission. However, users may print, download, or email articles for individual use. 